\title{
Matching Globular Cluster Models to Observations
}

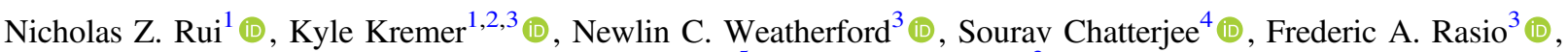 \\ Carl L. Rodriguez ${ }^{5}$ (D), and Claire S. $\mathrm{Ye}^{3}$ (D) \\ ${ }^{1}$ TAPIR, California Institute of Technology, Pasadena, CA 91125, USA; nrui@ caltech.edu \\ 2 The Observatories of the Carnegie Institution for Science, Pasadena, CA 91101, USA \\ ${ }^{3}$ Center for Interdisciplinary Exploration \& Research in Astrophysics (CIERA) and Department of Physics \& Astronomy, Northwestern University, Evanston, IL \\ 60208, USA \\ ${ }^{4}$ Tata Institute of Fundamental Research, Homi Bhabha Road, Navy Nagar, Colaba, Mumbai 400005, India \\ ${ }^{5}$ Department of Physics, Carnegie Mellon University, Pittsburgh, PA 15213, USA \\ Received 2021 January 21; revised 2021 March 5; accepted 2021 March 8; published 2021 May 10
}

\begin{abstract}
As ancient, gravitationally bound stellar populations, globular clusters represent abundant, vibrant laboratories, characterized by high frequencies of dynamical interactions, coupled to complex stellar evolution. Using surface brightness and velocity dispersion profiles from the literature, we fit 59 Milky Way globular clusters to dynamical models from the CMC Cluster Catalog. Without performing any interpolation, and without any directed effort to fit any particular cluster, 26 globular clusters are well matched by at least one of our models. We discuss in particular the core-collapsed clusters NGC 6293, NGC 6397, NGC 6681, and NGC 6624, and the non-corecollapsed clusters NGC 288, NGC 4372, and NGC 5897. As NGC 6624 lacks well-fitting snapshots on the main CMC Cluster Catalog, we run six additional models in order to refine the fit. We calculate metrics for mass segregation, explore the production of compact object sources such as millisecond pulsars, cataclysmic variables, low-mass X-ray binaries, and stellar-mass black holes, finding reasonable agreement with observations. In addition, closely mimicking observational cuts, we extract the binary fraction from our models, finding good agreement, except in the dense core regions of core-collapsed clusters. Accompanying this paper are a number of python methods for examining the publicly accessible CMC Cluster Catalog, as well as any other models generated using CMC.
\end{abstract}

Unified Astronomy Thesaurus concepts: Globular star clusters (656); Computational methods (1965); Cataclysmic variable stars (203); X-ray binary stars (1811); Binary stars (154); Stellar-mass black holes (1611); Stellar dynamics (1596)

\section{Introduction}

Some of the oldest known structures, globular clusters (GCs), are gravitationally bound stellar populations located in galactic halos which formed $\sim 13 \mathrm{Gyr}$ ago, in the early universe (Hut \& Heggie 2003). The pervasiveness and rich dynamical activity within globular clusters make them excellent sandboxes in which to study an abundance of stellar exotica, including X-ray binaries, radio millisecond pulsars, and gravitational wave sources (Hui et al. 2010; Ivanova et al. 2010; Bae et al. 2014; Kremer et al. 2018a, 2018b; Ye et al. 2019).

GCs are large $\left(N \gtrsim 10^{5}-10^{6}\right)$ self-gravitating systems of objects with a large range of masses, for which the dynamics are both complicated and critical to the formation and subsequent evolution of the cluster. In recent years, some authors (Hut et al. 1992; Chatterjee et al. 2013; Kremer et al. 2020) have demonstrated that GCs which exhaust their supply of black holes undergo runaway core stellar density ("core collapse") which is only stabilized by dynamical interactions between binaries ("binary burning"). Core collapse is characterized observationally by a highly compact, bright core, with a surface brightness profile which appears to constantly increase toward the GC's center, whereas the lack of core collapse is associated with a GC core with roughly flat surface brightness. Today, around one-fifth of observed GCs in the Milky Way display the extreme central concentration in surface brightness characteristic of core collapse (Harris 1996, 2010 edition).
Although it is in principle the most trustworthy method for GC dynamical modeling, direct $N$-body integration is extremely computationally expensive (requiring, e.g., a year of supercomputing time for $N \simeq 10^{6}$ particles, in Wang et al. 2016), thus restricting its wide-scale application to star clusters with $N \lesssim 10^{4}-10^{5}$ (e.g., Zonoozi et al. 2011, 2014), or requiring approximate ad hoc scalings with $N$ to realistic GC sizes (Aarseth \& Heggie 1998; Baumgardt 2001).

Fortuitously, the introduction of more efficient methods, such as the Monte Carlo algorithm, first introduced by Hénon (Hénon 1971; Stodolkiewicz 1986; Giersz 1998; Joshi et al. 2000), has made possible the simulation of comprehensive GC model grids on realistic time frames (Rodriguez et al. 2016). This development has kicked off extensive recent work on GC dynamics (e.g., Joshi et al. 2001; Fregeau et al. 2003; Fregeau \& Rasio 2007; Chatterjee et al. 2010; Giersz \& Heggie 2011; Umbreit et al. 2012; Giersz et al. 2013, 2015), and specifically enables the analysis presented below. In this work, we examine GC models generated by Cluster Monte Carlo (CMC), a Hénon-style Monte Carlo code that computes the evolution of GCs under the assumption of spherical symmetry (Pattabiraman \& Umbreit 2013).

To complement the upcoming release of C. L. Rodriguez et al. (in preparation, 2021), we explore the most recent grid of Milky Way GC dynamical models, the CMC Cluster Catalog (Kremer et al. 2020), and present a procedure for determining a modern-day GC's location on the grid via its observed surface brightness and velocity dispersion profiles (SBPs and VDPs). We summarize the CMC Cluster Catalog in Section 2.1, 
Table 1

Summary of the Initial Cluster Parameters of the CMC Cluster Catalog

\begin{tabular}{lc}
\hline \hline Parameter & Values \\
\hline Initial number of stars $N\left(\times 10^{5}\right)$ & $2,4,8,16,32^{\mathrm{a}}$ \\
Virial radius $r_{v}(\mathrm{pc})$ & $0.5,1.0,2.0,4.0$ \\
Galactocentric distance $R_{g}(\mathrm{kpc})$ & $2,8,20$ \\
Metallicity $[\mathrm{M} / \mathrm{H}]$ & $-2,-1,0$ \\
\hline
\end{tabular}

Note.

${ }^{\text {a }}$ Due to computational expense, the grid only covers a subset of the permitted $r_{v}, R_{g}$, and $[\mathrm{M} / \mathrm{H}]$ values for $N=3.2 \times 10^{6}$.

and the fitting procedure in Section 2.2. For concreteness, we specifically examine six of the GCs well fit by this procedure, i.e., the core-collapsed clusters NGC 6293, 6397, and 6681 (Sections 3.1-3.3), and the non-core-collapsed clusters NGC 288, 4372, and 5897 (Sections 3.4-3.6), which are all well fit by the CMC Cluster Catalog as is. We also consider NGC 6624, an interesting, high-metallicity cluster which is not captured initially on the $\mathrm{CMC}$ Cluster Catalog proper, and extend the model grid with additional $\mathrm{CMC}$ models to obtain a good fit (Section 3.7). For these clusters, we consider exotic binary and millisecond pulsar populations, cluster masses and mass-to-light ratios (Section 4.1), binary fractions (Section 4.2), mass segregation (Section 4.3), and black holes (Section 4.4). Accompanying this work is a set of publicly available python functions and simulation properties needed to reproduce this analysis $^{6}$ (Rui et al. 2021).

\section{Methods}

Here, we outline the methods used to compare our cluster models to the observed data of Milky Way GCs. In Section 2.1, we broadly summarize the CMC Cluster Catalog, and, in Section 2.2, we describe the procedure for extracting the simulated surface brightness and velocity dispersion, and using these to fit the observed data. In Section 2.3, we detail criteria for identifying various stellar exotica in our models.

\subsection{Model Grid}

The CMC Cluster Catalog comprises 148 models, spanning a realistic and comprehensive range of initial virial radii, tidal radii, metallicities, and masses (Table 1), integrated via CMC. Within CMC, stellar evolution is modeled using the SINGLE-STAR EVOLUTION (SSE, Hurley et al. 2000) and BINARY-STAR EVOLUTION (BSE, Hurley et al. 2002) algorithms, updated to include the most current prescriptions for compact object formation (see, e.g., Breivik et al. 2020). These prescriptions describe the evolution of stars/stellar objects through various evolutionary stages, distinguished in the code by "stellar type" (see Section 1 of Hurley et al. 2000 for a list of stellar types and discussion). CMC also incorporates the physics of three/four-body encounters, integrated using the FEWBODY package (Fregeau et al. 2004; Fregeau \& Rasio 2007), and updated to include post-Newtonian terms (Rodriguez et al. 2018). Our models assume that GCs experience a constant tidal field throughout their lifetimes. Of course, in general, GCs undergo complicated orbits, characterized by periapse passages which may affect their dynamics in a nonlinear fashion, and there is evidence to suggest that the galactic potential has itself

\footnotetext{
6 https://github.com/NicholasRui/cmctoolkit
}

varied over time in the history of the Milky Way (e.g., Kruijssen et al. 2019). Further exploration of the effects of such time-dependent tidal fields falls beyond the scope of this work.

At various snapshots in time, separated by multiples of the estimated dynamical time of the cluster, CMC writes a catalog of stellar and kinematic properties for all stars in the cluster. In this study, we are primarily interested in cluster models similar to the old GCs observed in the Milky Way; we therefore restrict our attention to snapshots for which $t>10 \mathrm{Gyr}$ (all models are run to $t \approx 14 \mathrm{Gyr}$, or until dissolution), of which there are 7537 throughout the entire $\mathrm{CMC}$ Cluster Catalog.

In general, the parameters most germane to dynamical structure are the initial number of stars, $N$, and the virial radius, $r_{v}$. The galactocentric distance, $R_{g}$, is most impactful through its influence at the outskirts of the cluster as it defines the tidal radius, while the metallicity, $Z$, primarily influences stellar evolution. Moreover, both $R_{g}$ and $Z$ are more easily estimated empirically than $N$ and $r_{v}$, which often change dramatically over the course of a cluster's lifetime (e.g., Kremer et al. 2020). We therefore limit our fitting procedure for each cluster to only those models where $R_{g}$ and $Z$ are closest (in linear and logarithmic scales, respectively) to their observed present-day values, as reported by Baumgardt et al. (2019b), and Harris (1996, 2010 edition), respectively. Hence, for any individual cluster, we only optimize over $N$ and $r_{v}$. For simplicity, a constant tidal radius is assumed, although we caution that GC orbits in the Galaxy generally induce time-dependent tidal forces (including possible close pericenter passages). Furthermore, the modern-day distance of a GC to the Galactic center may not be representative of the average tidal force (Baumgardt et al. 2019b).

\subsection{Synthetic Observables and Cluster Fitting}

In order to match an observed GC with a best-fit model on our grid, we identify models whose dynamical properties most closely match the observed cluster features. The most direct dynamical observables of a GC are its surface brightness and velocity dispersion profiles; we therefore extract a simulated SBP and VDP from each model snapshot for comparison to the corresponding observed profiles.

Since GCs are observed only in projection on the sky, we project our simulated stellar positions and velocities onto a two-dimensional plane by assuming spherical symmetry. In particular, a star with a three-dimensional radius, $r$, has a probability $p(a, b ; r)$ of lying within projected radial distances $d=a$ and $d=b>a$, given by

$$
p(a, b ; r)=\sqrt{1-\min (a, r)^{2} / r^{2}}-\sqrt{1-\min (b, r)^{2} / r^{2}} .
$$

We calculate the surface brightness, $\Sigma_{V}(a, b)$, and onedimensional velocity dispersion, $\sigma_{v}(a, b)$, for 80 logarithmically spaced bins, with an inner bin of $10^{-3} \mathrm{pc}$, and an outer bin given by the maximum radial position of a star in the catalog. For $\sigma_{v}(a, b)$, we only include evolved bright stars (SSE/BSE with stellar types 2-9), so as to mimic the use of bright stars in real VDP measurements (e.g., Kamann et al. 2017; Ferraro et al. 2018b).

Given the two-dimensional distribution described by Equation (1), the average $V$-band surface brightness, $\Sigma_{V}(a, b)$, in a projected radial bin bounded by $d \in(a, b]$ can be 
calculated as

$$
\begin{aligned}
\Sigma_{V}(a, b)= & \\
& -2.5 \log _{10}\left(\frac{100 \mathrm{pc}^{2} \operatorname{arcsec}^{2}}{\pi\left(b^{2}-a^{2}\right)} \sum_{i} \frac{p\left(a, b ; r_{i}\right)}{10^{M_{V, i} / 2.5}}\right)+A_{V},
\end{aligned}
$$

where $r_{i}$ and $M_{V, i}$ are the three-dimensional radial distance and absolute $V$-band magnitude of the $i$ th star in the simulation, respectively, and $A_{V}$ is the $V$-band extinction. For simplicity, all stars are assumed to be blackbodies, which should reasonably approximate their actual magnitudes, particularly for more massive stars, where molecular lines are less prominent. Stellar magnitudes thus take the form

$$
M_{V, i}=-2.5 \log _{10}\left(\frac{R_{i}^{2}}{(10 \mathrm{pc})^{2} F_{\mathrm{ZP}, \lambda}} \frac{\int f\left(\lambda, T_{i}\right) \mathcal{T}(\lambda) d \lambda}{\int \mathcal{T}(\lambda) d \lambda}\right),
$$

where $F_{\mathrm{ZP}, \lambda}=3.57453 \times 10^{-9} \mathrm{erg} \mathrm{cm}^{-2} \mathrm{~s}^{-1} \AA^{-1}$ is the zeropoint spectral flux, $f(\lambda, T)$ is the wavelength-space Planck distribution for temperature $T$, and $\mathcal{T}(\lambda)$ is the transmission function for the filter (Casagrande \& VandenBerg 2014). Photometric magnitudes are derived using the generic Johnson $V$-band filter function and Vega zero point from the SVO Filter Profile Service, a public repository for astronomical filter parameters (Rodrigo \& Solano 2013; Rodrigo et al. 2020). The $V$-band extinction, $A_{V}$, of a $\mathrm{GC}$ is computed using the standard Cardelli et al. (1989) extinction law as $A_{V}=3.1 \times$ $E(B-V)$, where $E(B-V)$ is taken from the Harris $(1996,2010$ edition) catalog. While the blackbody approximation deviates significantly for cool $\mathrm{M}$ dwarfs, which have significant molecular absorption lines (Allard et al. 1994; Baraffe et al. 1995), the approximation is a reasonable estimate for brighter and hotter stars, whose continuum emission dominates the profile. Equation (3) is also applied in Section 4.2 to select binaries, although the cut is restricted to relatively bright main sequence stars, where the blackbody approximation should be expected to hold.

The velocity dispersion, $\sigma_{v}(a, b)$, in the same radial bin is given by

$$
\sigma_{v}(a, b)=\sqrt{\frac{\sum_{i}\left|v_{i}\right|^{2} p\left(a, b ; r_{i}\right)}{3 \cdot \sum_{i} p\left(a, b ; r_{i}\right)}},
$$

where $\boldsymbol{v}_{i}$ is the three-dimensional velocity vector of the $i$ th star. This expression for $\sigma_{v}(a, b)$ assumes an isotropic velocity dispersion. Although, in principle, the tangential and radial components of the velocity dispersion may differ, in most cases the ratio of the two is very close to one, particularly for cases near to a cluster's center (Watkins et al. 2015). Furthermore, although some have claimed detection of coherent rotation within many GCs (e.g., Kamann et al. 2017), this subtle behavior is not captured in our models, and we do not consider departures from spherical symmetry in this work. Such rotation is, in any case, usually much smaller than the velocity dispersion, and would not be expected to change it significantly, especially in the central regions of the GC, where CMC is expected to be most accurate. While spherical asymmetry is the subject of very interesting work (see, e.g., Gieles et al. 2021, where measurements of Palomar 5's tidal tails are used in fitting), it lies beyond the scope of this work.

Here, we assess the relative likelihood that a given $\mathrm{CMC}$ model fits observed SBPs/VDPs by computing $\chi^{2}$ statistics between this data and linearly interpolated model SBPs/VDPs (Heggie \& Giersz 2008, 2014; Giersz \& Heggie 2009, 2011; Kremer et al. $2018,2019)$. The fitness of a model with a given $\mathrm{GC}$ is assessed using $\tilde{\chi}_{\mathrm{SBP}}^{2}=\chi_{\mathrm{SBP}}^{2} / N_{\mathrm{SBP}}$ and $\tilde{\chi}_{\mathrm{VDP}}^{2}=\chi_{\mathrm{VDP}}^{2} / N_{\mathrm{VDP}}$, the $\chi^{2}$ statistics between the model SBP/VDP, and the observations, normalized by the number of observational data points. For a given GC, we consider well-fitting snapshots to have the fitting heuristic $s \equiv \max \left(\tilde{\chi}_{\mathrm{SBP}}^{2}, \tilde{\chi}_{\mathrm{VDP}}^{2}\right)<10$. As such, to be a "good fit" to the data, a snapshot must be a reasonably good fit to both the SBP and VDP. For diagnostic reasons, we also report the best-fitting snapshot of each cluster $\tilde{\beta}_{\mathrm{SBP}}^{2}$ and $\tilde{\beta}_{\mathrm{VDP}}^{2}$, defined as the reduced $\chi^{2}$ sums with terms weighted by the sign of the residual. These statistics parameterize the extent to which a given snapshot overestimates or underestimates a cluster's surface brightness or velocity dispersion, and are included to guide the creation of future models in order to better fit particular observed GCs.

The SBPs are taken from ground-based observations by Trager et al. (1995). ${ }^{7}$ While other data sets, such as the Noyola \& Gebhardt (2006) SBPs for 38 GCs, may better probe the surface brightnesses of GC cores, particularly with respect to core-collapsed clusters, we opt to exclude this data, in order to avoid assigning ad hoc relative weights between the Trager and Noyola profiles. The VDPs are taken from the radial velocity measurements of Kamann et al. (2017), Ferraro et al. (2018b), and Baumgardt \& Hilker (2018), as well as the proper motion measurements of Watkins et al. (2015), and Baumgardt et al. (2019b). The VDPs measured using radial velocities and proper motions are generally observed to be consistent with one another; cluster-VDP uncertainties across all data sets are therefore taken as reported, without any rescaling or homogenization, and we fit to a combination of these VDPs for maximal constraint.

As Trager et al. (1995) do not calculate formal uncertainties on their SBP measurements, but instead provide a Chebyshev polynomial fit, and coarse quality weights, $w_{i}$, for each data point, we follow the procedure adopted in McLaughlin \& van der Marel (2005) in order to estimate uncertainties. In particular, we assume that the measurement uncertainties are inversely proportional to $w_{i}$, and that the third-order Chebyshev polynomial fits have $\tilde{\chi}^{2}=1$ exactly. We then estimate the uncertainty of the $i$ th data point to be $\delta \Sigma_{V}=\delta \Sigma_{V, 0} / w_{i}$, where $\delta \Sigma_{V, 0}$ is estimated separately for each cluster as $\delta \Sigma_{V, 0}=$ $\sqrt{\left(N_{\mathrm{SBP}}-4\right)^{-1} \sum_{i=1}^{N} w_{i}^{2} e_{\Sigma_{V}, i}^{2}}$, where $e_{\Sigma_{V}, i}$ is the surface brightness residual of the $i$ th data point with respect to the Chebyshev polynomial fit. Since this effectively discards observations for which Trager et al. (1995) assign $w_{i}=0$, we omit these points when normalizing $\tilde{\chi}_{\mathrm{SBP}}^{2}$ by $N_{\mathrm{SBP}}$. As the publicly available SBP for NGC 2419 appears to be multi-valued, we restrict our profile to data sets given in Trager et al. (1995), following the branch shown in the plot in their paper, and we estimate the SBP uncertainties using these points alone.

\footnotetext{
7 As the publicly available SBP for NGC 2419 appears to be multi-valued, we restrict this profile to data sets in Trager et al. (1995), which follow the branch shown in the plot in their paper, and estimate the SBP uncertainties using these points alone.
} 

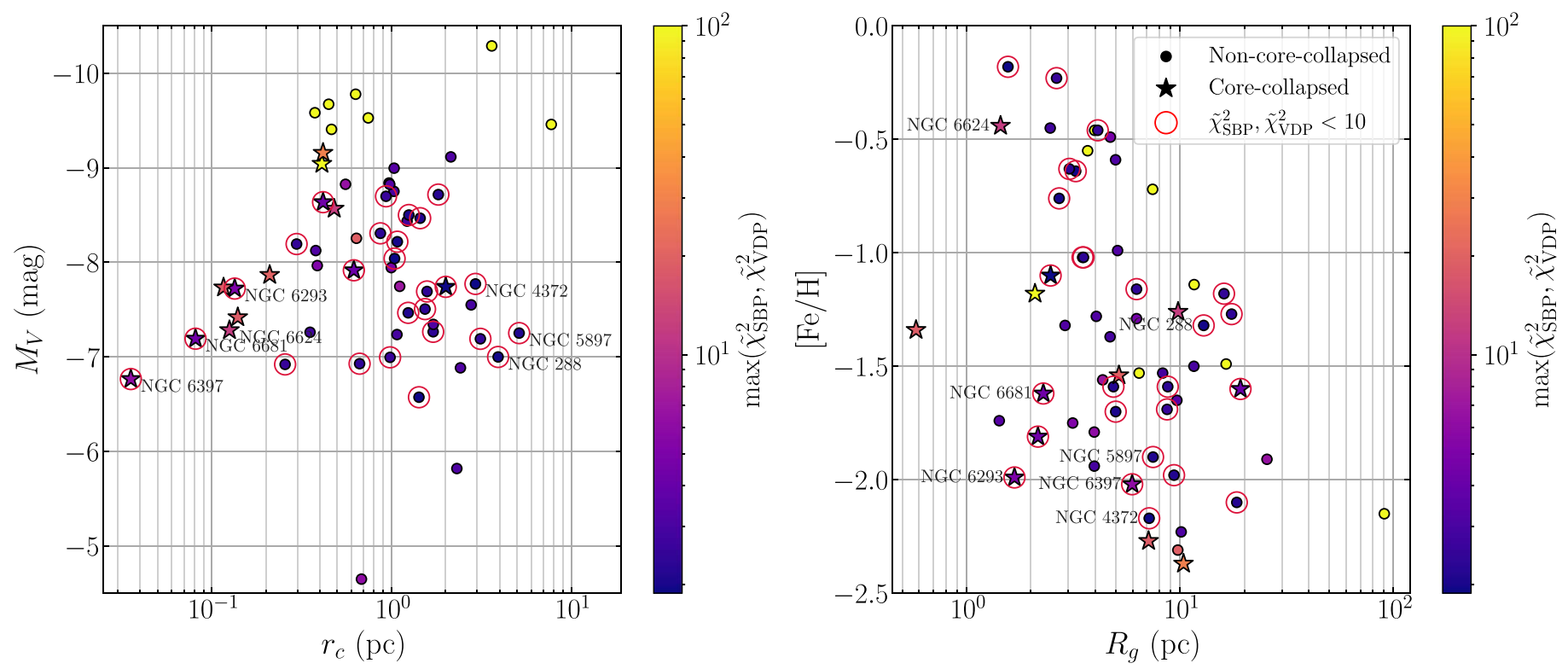

Figure 1. GCs for which best-fit CMC Cluster Catalog models are identified, plotted in $r_{c}-M_{V}$ (left) and $R_{g}-[\mathrm{Fe} / \mathrm{H}]$ (right) space. GCs are color coded based on $s=\max \left(\tilde{\chi}_{\mathrm{SBP}}^{2}, \tilde{\chi}_{\mathrm{VDP}}^{2}\right)$, with some GCs saturating the color bar from above. Clusters considered to be "well fit" $(s<10)$ are circled in red, and clusters specifically discussed in the text are labeled.

Figure 1 shows the GCs for which both the SBP and VDP are sampled with at least five points, in both the core radiusbrightness $\left(r_{c}-M_{V}\right)$ and galactocentric distance-metallicity $\left(R_{g}-[\mathrm{Fe} / \mathrm{H}]\right)$ planes, with $M_{V}, r_{c}$, and $[\mathrm{Fe} / \mathrm{H}]$ taken from Harris (1996, 2010 edition), and $R_{g}$ from Baumgardt et al. (2019b). Points are color coded by the fitting statistic, $s$, with well-fit clusters circled. Despite the wide range of Milky Way GC properties, we are able to satisfactorily fit a wide range of clusters across the observed parameter space. As expected, well-fit clusters are concentrated at lower brightnesses, specifically at dimmer $M_{V} \gtrsim-9.5$, indicating a lack of grid coverage at larger masses. Unsurprisingly, the quality of the fit does not obviously correlate with present-day galactocentric distance or metallicity, as the bulk cluster dynamics are less sensitive to these parameters.

\subsection{Identification of Stellar Exotica}

In the sections below, we examine best-fitting models for seven specific GCs, during which we make comparisons to the observed population of low-mass X-ray binaries, millisecond pulsars, and cataclysmic variables. Although we do not use these stellar exotica as factors in our goodness-of-fit measurements, due to their large uncertainties, we can use the rough numbers as guidelines to further constrain and explore our models.

An X-ray binary $(\mathrm{XRB})$ is a mass-transferring binary where the donor, typically a main sequence star, accretes onto a compact object, either a neutron star or a black hole. While short-lived high-mass X-ray binaries (with OB-type donors) dominate the X-ray sources in young, star-forming populations, low-mass X-ray binaries (LMXBs) are believed to form in the dense cores of GCs (Pooley et al. 2003; Fabbiano 2006). In our models, we consider as XRBs any main sequence star (SSE stellar type $0-1$ ) in a mass-transferring binary with a neutron star or black hole. Our models contain characteristically between zero and a few XRBs.

One possible outcome of a disrupted LMXB is a millisecond pulsar. Millisecond pulsars (MSPs) are rapidly rotating pulsars with periods on the order of milliseconds. Unlike standard pulsars, which are young neutron stars rotating fast enough to beam, MSPs are older neutron stars which have been "recycled" by accretion from a companion. Whereas the former have periods $P \simeq 0.1-3 \mathrm{~s}$, mass transfer onto the latter spins MSPs up to periods as small as $1.5 \mathrm{~ms}$ (Lorimer 2008). Such objects are expected to be formed at an enhanced rate in the high-density environments at the center of GCs, particularly in core-collapsed clusters with very few black holes (Ye et al. 2019). In our models, we identify MSPs as neutron stars with periods $P<10 \mathrm{~ms}$.

Cataclysmic variables (CVs) are usually low-mass main sequence stars undergoing mass transfer onto a white dwarf via Roche-lobe overflow. As their name suggests, they are characterized by large, often rapid flux variability, in some cases undergoing violent eruptions in the form of novae or dwarf novae (Robinson 1976). GCs are believed to harbor significant $\mathrm{CV}$ populations, which may help to guide studies of their evolution, as well as their possible role as $\mathrm{SNe}$ Ia progenitors (Ivanova et al. 2006; Knigge 2012; Maoz et al. 2014). Similarly to XRBs, we identify CVs as SSE stellar type 0 stars undergoing mass transfer onto white dwarfs. Our models contain a wide range of $\mathrm{CVs}$, spanning from a few to $\sim 100$ CVs. Interestingly, given that most CVs originate from primordial binary progenitors, the number of $\mathrm{CVs}$ in a $\mathrm{GC}$ actually correlates inversely with its central density. This scaling is in contrast to that of other objects (e.g., MSPs), whose formation is predominantly dynamical.

Blue straggler stars are unusually bright/blue main sequence stars which have been rejuvenated, either via accretion from another star (e.g., Chen \& Han 2004), or a collision at some point in the GC's history (e.g., Glebbeek et al. 2008). With links to both standard binary evolution and cluster dynamics, the radial distribution of blue stragglers within GCs has gained significant attention as a possible tracer of a cluster's dynamical history (e.g., Ferraro et al. 2012, 2018a). We leave the modeling of these interesting sources to a future work. 


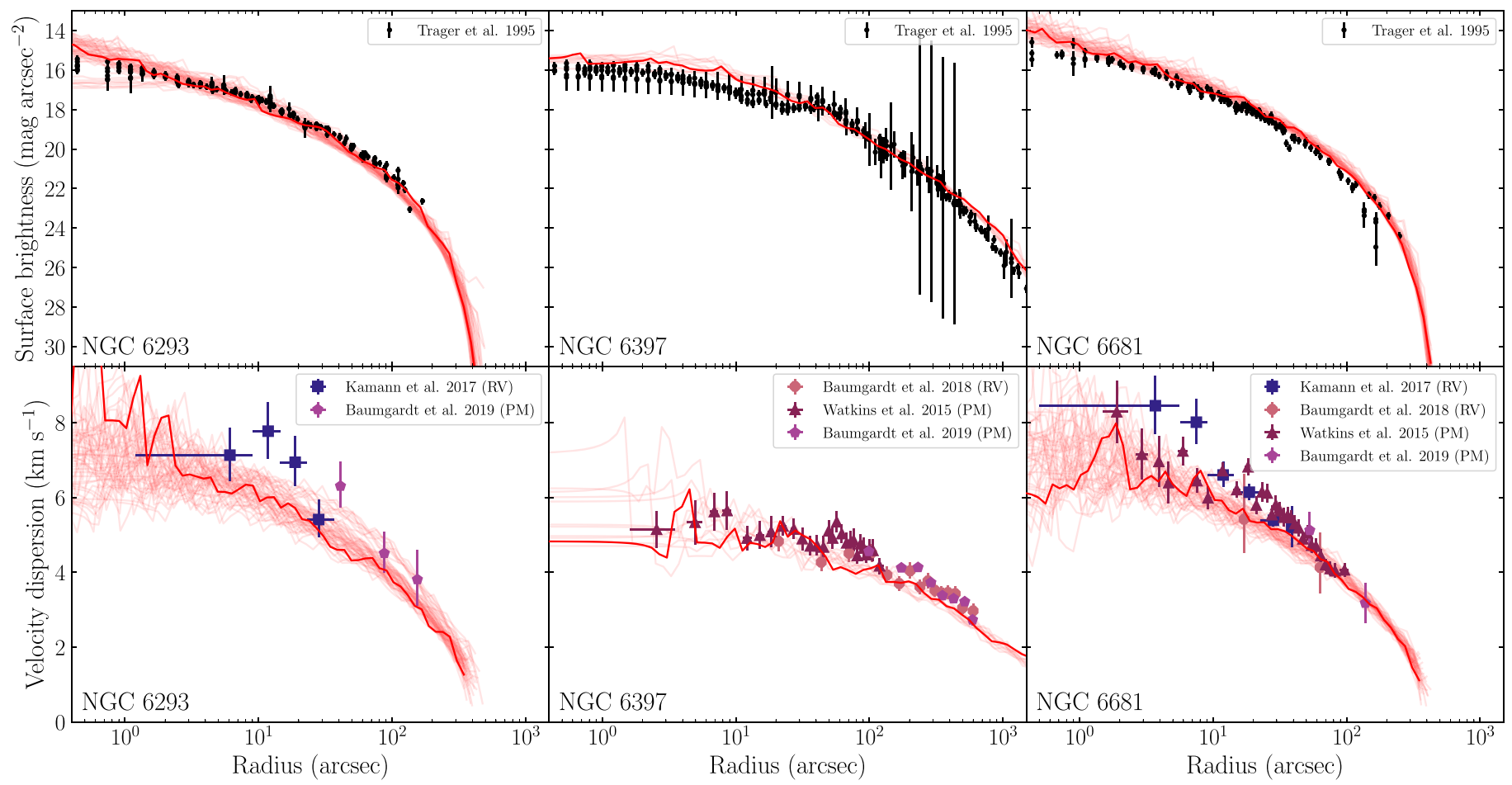

Figure 2. SBPs and VDPs, together with best-fitting models for the core-collapsed clusters NGC 6293, NGC 6397, and NGC 6681. The best-fit profile is shown as an opaque red curve, and the SBPs and VDPs of well-fitting snapshots $(s<10)$ are shown as translucent red curves.

\section{Fitting Clusters to Observations}

Using the procedure described in Section 2.2, we identify best fits for 59 Milky Way GCs in which $N_{\mathrm{SBP}}, N_{\mathrm{VDP}} \geqslant 5$ (see Appendix). We obtain at least one "well-fitting" snapshot $(s<10)$ for $26 \mathrm{GCs}$, including the core-collapsed clusters NGC 6293, 6397, and 6681 (Figures 2 and 3), and non-corecollapsed clusters NGC 288, 4372, and 5897 (Figures 4 and 5). We emphasize that these best-fitting snapshots exist on the CMC cluster Catalog as-is, without interpolation, or any directed effort to fit any particular cluster. A more precise representation of a particular $\mathrm{GC}$ requires the creation of new CMC models. These GCs cover a range of dynamical states, masses, distances, and metallicities, and allow us to benchmark our model predictions with a variety of cluster properties. The non-core-collapsed clusters selected for further examination have large core radii (as seen from Earth) - together with the core-collapsed clusters of interest, these GCs allow us to explore the full range of realistic values of $r_{c}$. However, we note that some GCs with intermediate core radii (e.g., NGC 6352) also fit well with some models in the CMC Cluster Catalog, as is apparent in Figure 1.

In addition, in order to demonstrate the potential to straightforwardly supplement the CMC Cluster Catalog to fit new clusters, and to present one example of a model refinement for a specific GC, we also extend the model grid in Section 3.7 to fit NGC 6624, an interesting high-metallicity cluster, known for its high-energy emission and large number of recorded millisecond pulsars.

\subsection{NGC 6293}

Reaching about $0.5 \mathrm{kpc}$ at perigalacticon, NGC 6293 is a low-metallicity, core-collapsed GC which lies very close to the Galactic center-an intense tidal environment which spatially flattens the cluster's shape (Chen \& Chen 2010;
Baumgardt et al. 2019b). Within the CMC Cluster Catalog, we locate 59 well-fitting snapshots from two core-collapsed models, N8-RV1-RG2-Z0.01 (8 snapshots), and N8-RV0.5-RG2Z0.01 (51 snapshots), both with an initial $N=8 \times 10^{5}$. While the SBPs are generally matched quite well within the cores, some snapshots slightly overestimate the core brightness, and some underestimate it. As such, well-fitting snapshots should produce predictions for cluster properties (e.g., total mass) which "surround" their true values. While all well-fitting snapshots underestimate the VDP somewhat, observational uncertainties are comfortably large enough to be consistent with predictions.

NGC 6293 is associated with at least one soft X-ray source (XTE J1709-267, Jonker et al. 2003). Of the 59 well-fitting snapshots, 54 snapshots do not have any XRBs, and five snapshots contain a single XRB (three from the $r_{v}=1 \mathrm{pc}$ model $\mathrm{pc}$, and two from the $r_{v}=0.5 \mathrm{pc}$ model). Furthermore, all of these XRBs have low-mass donors with SSE stellar type 0 $\left(M \lesssim 0.7 M_{\odot}\right)$. Despite possible observational biases, and incompleteness on the total number of XRBs in the cluster (especially for a cluster near the Galactic Center), our models appear consistent with the capacity of NGC 6293 to produce a small number of X-ray sources.

\subsection{NGC 6397}

NGC 6397 is a nearby $(D=2.44 \pm 0.04 \mathrm{kpc}$, Baumgardt et al. 2019b) metal-poor, core-collapsed GC, whose close proximity has attracted significant study of its white dwarf and low-mass stellar populations (Paresce et al. 1995; Cool et al. 1996; Taylor et al. 2001; Hansen et al. 2007). In addition, NGC 6397 has 15 known CV candidates, a quiescent LMXB (qLMXB), and 1-2 MSPs (Cool et al. 1995; Grindlay et al. 2001; Cohn et al. 2010; Dieball et al. 2017). We locate 11 wellfitting snapshots from the model, N4-RV1-RG8-Z0.01. While all 

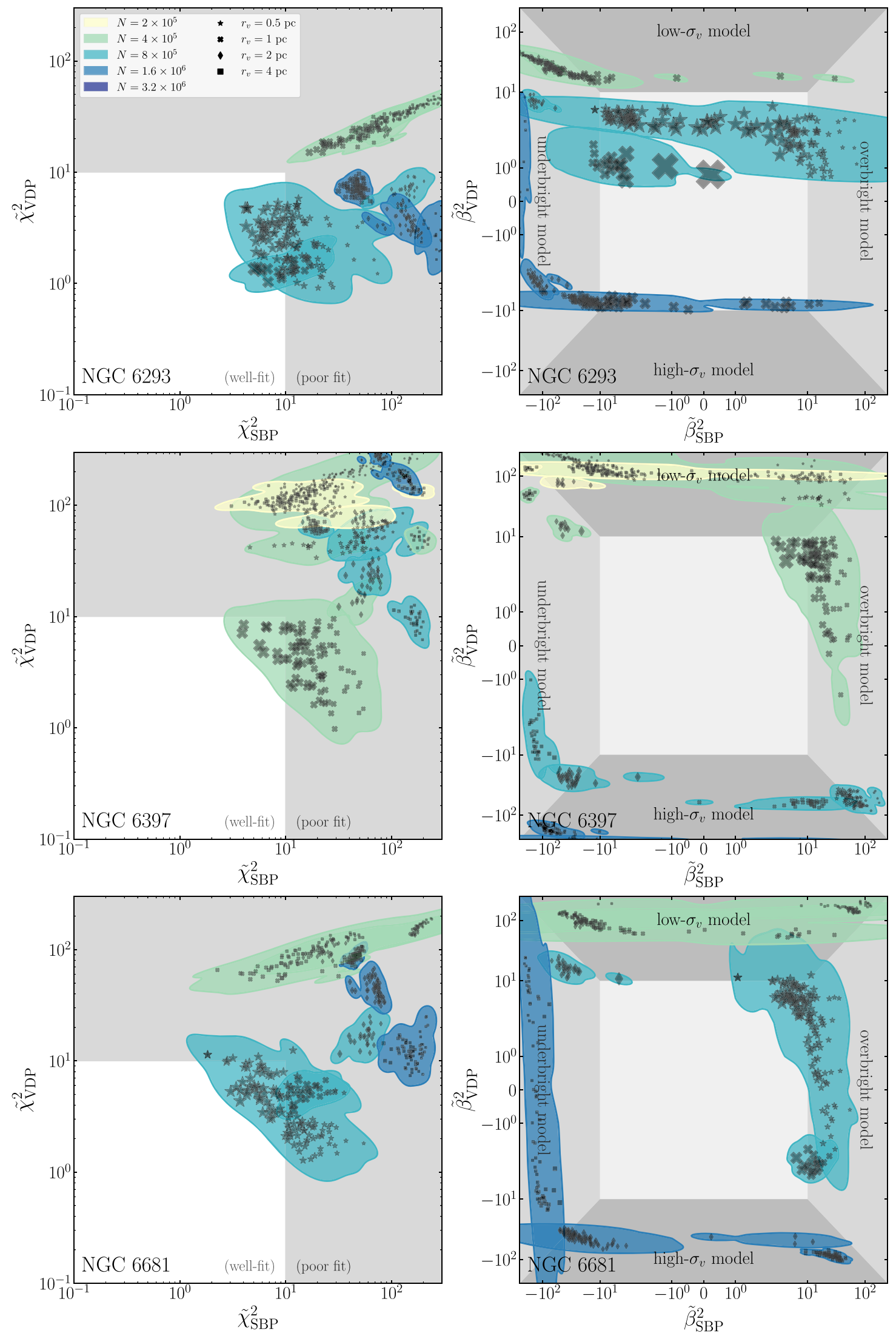

Figure 3. Left: plots of $\tilde{\chi}_{\mathrm{SBP}}^{2}$ and $\tilde{\chi}_{\mathrm{VDP}}^{2}$ for simulated SBPs and VDPs of snapshots where $t \geqslant 10$ Gyr vs. NGC 6293, NGC 6397, and NGC 6681, which are all corecollapsed today. The colorful, shaded regions are level curves of kernel density estimates for individual models to guide the eye, and are colored by the initial number of stars in the simulation. We consider "good fits" to be given by $s<10$. Right: as for the plots on the left, except for $\tilde{\beta}_{\mathrm{SBP}}^{2}$ and $\tilde{\beta}_{\mathrm{VDP}}^{2}$. These parameters function as estimators with respect to how much a model overestimates the SBP or VDP of a cluster. Points far from the origin are reduced in size for clarity. 


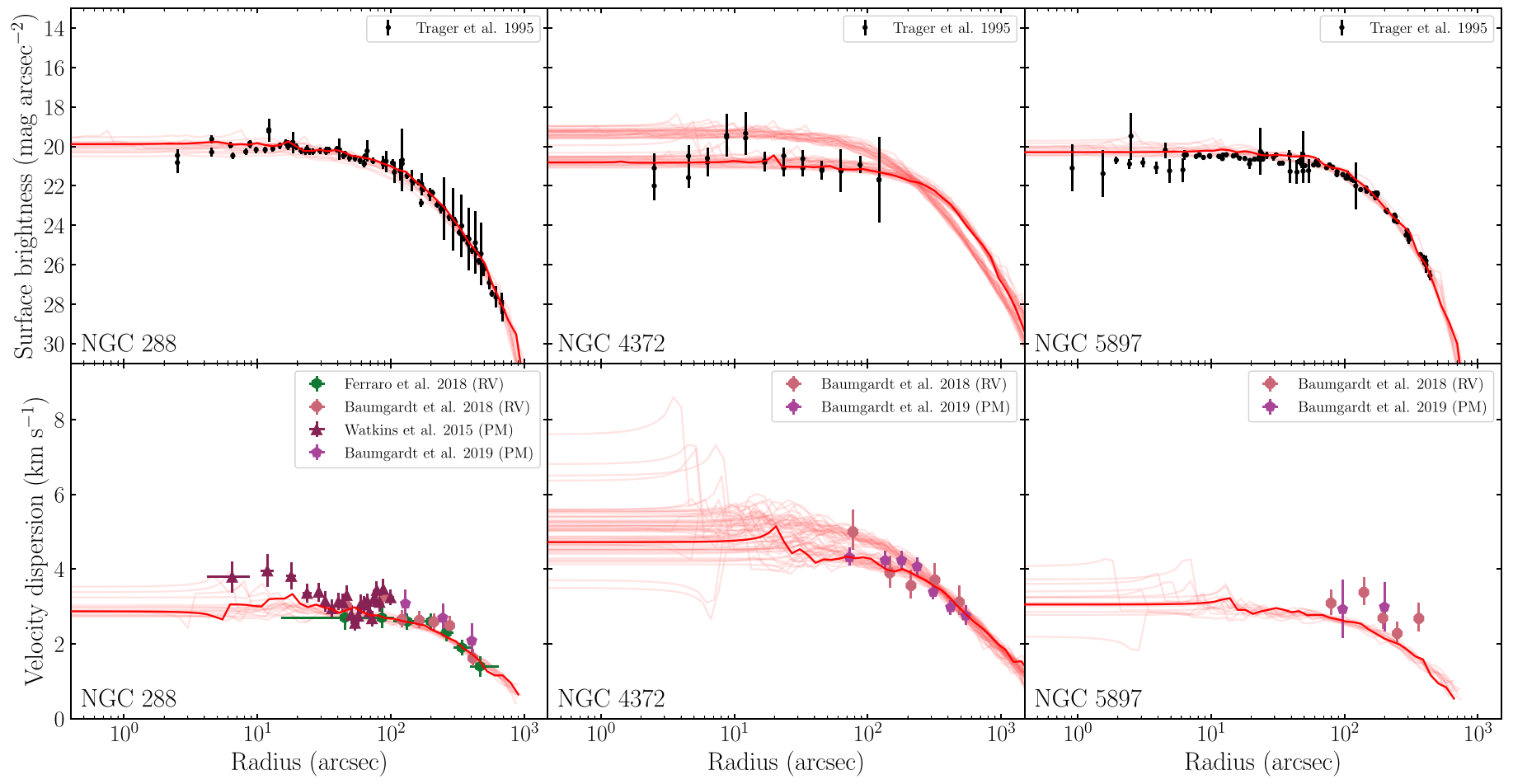

Figure 4. SBPs and VDPs as shown in Figure 2, but for non-core-collapsed clusters NGC 288, NGC 4372, and NGC 5897.

of these snapshots tend to overestimate the surface brightness, and underestimate the velocity dispersion somewhat, they do so within our tolerances. By eye, it appears that the slight overestimation of the SBP occurs primarily at $\approx 5 \mathrm{pc}$, where the model is slightly brighter than the data.

The well-fitting snapshots for this cluster each have between 11 and $13 \mathrm{CVs}$. In reasonable agreement with observations, these snapshots also show between 1 and 2 XRBs each (all of which have $M \lesssim 0.7 M_{\odot}$ donors). Finally, these snapshots all contain two MSPs. Of course, while these small numbers should not be taken as precise predictions, they provide a measure of reassurance that our models generate these populations in reasonable numbers.

\subsection{NGC 6681}

NGC 6681 (M70) is a core-collapsed cluster, which has occasionally been used to derive distortion solutions for instruments operating in the far-ultraviolet (e.g., Sohn 2018). Like NGC 6293, NGC 6681 lies quite close to the Galactic center $(R \sim 0.8 \mathrm{kpc}$, Baumgardt et al. 2019b), and observations also suggest some degree of tidal deformation (Han et al. 2017). We find 49 well-fitting snapshots from the corecollapsed model, N8-RV0.5-RG2-Z0.01. As this particular model also provides snapshots consistent with the SBP and VDP of NGC 6293, one can similarly view NGC 6681 as somewhat similar to NGC 6293 from a dynamical perspective. As with NGC 6293, all snapshots slightly underestimate the VDP, while remaining within tolerance in terms of observational uncertainties.

\section{4. $N G C 288$}

A relatively metal-rich, non-core-collapsed GC, NGC 288 garnered much attention in the 1990s and early 2000s, as its similar metallicities and distances to those of NGC 362 provided promising avenues for constraining the age difference between the two clusters (Green \& Norris 1990; Sarajedini \& Demarque 1990; Bellazzini et al. 2001). There is a large number of subsequent studies into its dynamics (e.g., Piatti 2018) and stellar populations (e.g., Roh et al. 2011). Within our model grid, we identify 16 well-fitting snapshots from a large $r_{v}=4$ model, N4-RV4-RG8-Z0.1. Both the SBPs and VDPs from these snapshots fit the observations exceptionally well.

Using Chandra, Kong et al. (2006) reported between two and five possible $\mathrm{CVs}$ or other chromospherically active binaries within the half-mass-radius of NGC 288. Meanwhile, our models predict between 27 and $33 \mathrm{CVs}$. This discrepancy may be attributable to the high temporal variability in the activity of CVs, which could make quiescent accreting binaries difficult to detect, or to some other source of observational incompleteness.

\subsection{NGC 4372}

While Trager et al. (1995) do measure the $V$-band SBP for this cluster, they do not report data outside the 1.75 core radius reported by Harris (1996, 2010 edition). As such, the SBP neither contains the characteristic turnover in brightness, nor constrains particularly well the size of the cluster's core. Therefore, while the CMC Cluster Catalog includes 30 well-fitting snapshots each from the $N=8 \times 10^{5}$ models, N8RV4-RG8-Z0.01, and N8-RV2-RG8-Z0.01, the initial virial radius of the cluster is largely uncertain. Although the snapshots come from models with different initial $r_{v}$, their similar initial masses and dynamical states produce a relatively small spread in the predicted mass of the cluster. Kacharov et al. (2014) estimated a cluster mass $M=(2.0 \pm 0.5) \times 10^{5} M_{\odot}$, compatible with the estimated mass from snapshots at the $1 \sigma$ level. They also estimate a mass-to-light ratio of between 1.4 and 2.3 for this cluster, which is broadly compatible with predicted values for all seven of our clusters of interest (see Section 4.1). 

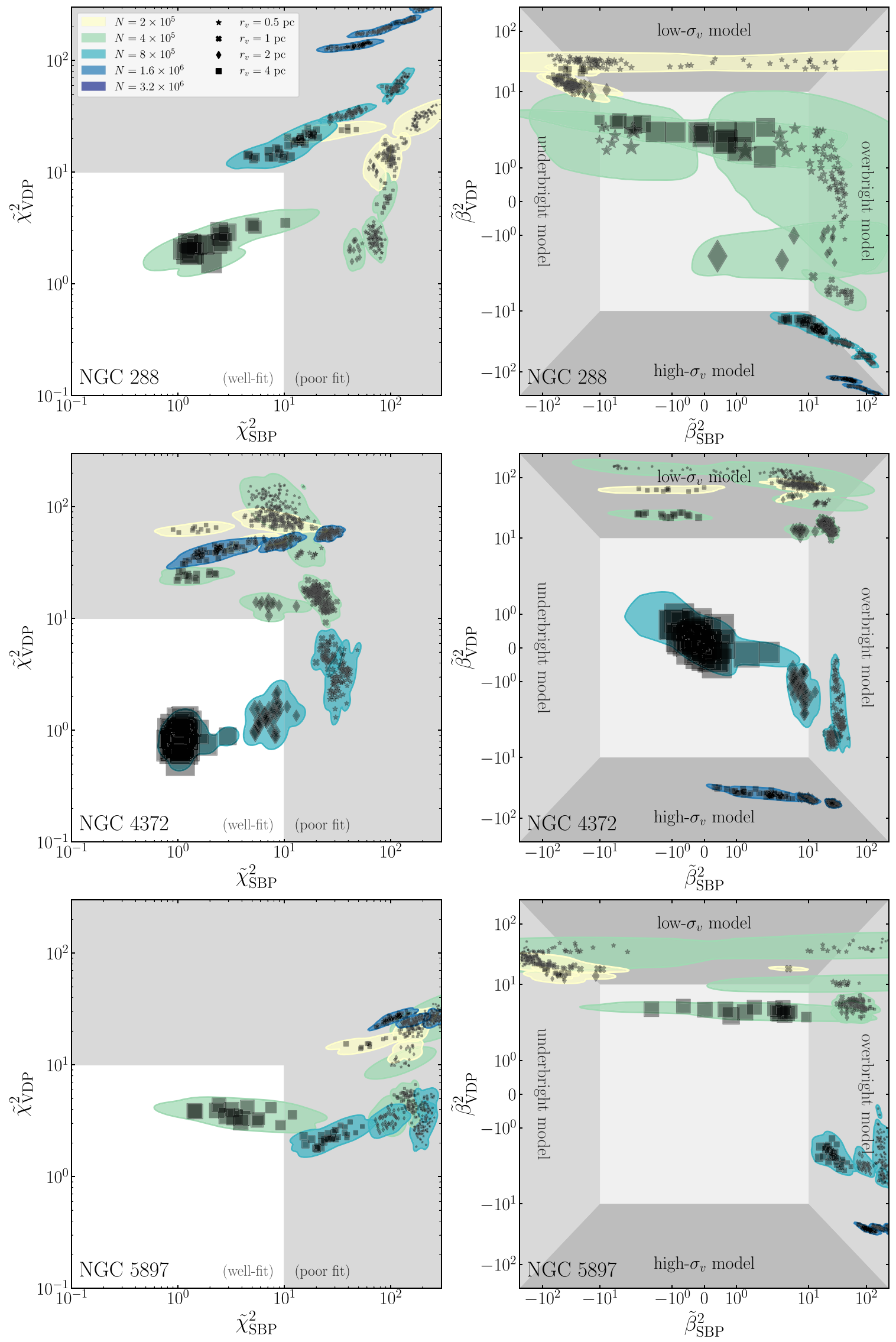

Figure 5. Values of $\tilde{\chi}_{\mathrm{SBP}}^{2}, \tilde{\chi}_{\mathrm{VDP}}^{2}, \tilde{\beta}_{\mathrm{SBP}}^{2}$, and $\tilde{\beta}_{\mathrm{VDP}}^{2}$ as in Figure 3, but for the non-core-collapsed clusters NGC 288, NGC 4372, and NGC 5897; well-fitting snapshots exist in all three cases here. 
Within NGC 4372, Kaluzny \& Krzeminski (1993) identify a candidate $\mathrm{CV}$, although atmospheric effects imply a high degree of incompleteness in the data. Using X-ray observations from XMM-Newton, Servillat et al. (2008) were unable to detect specific $\mathrm{CVs}$-although these were limited by X-ray luminosity - although they report unresolved emission, consistent with a population of $\sim 20 \mathrm{CVs}$. As our well-fitting snapshots come from two models with different initial $r_{v}$, we find a bimodal distribution, with modes approximately around 26 and $84 \mathrm{CVs}$, and with well-fitting snapshots having between 21 and 98. The lower and higher modes correspond to snapshots from the $r_{v}=2.0 \mathrm{pc}$ and $r_{v}=4.0 \mathrm{pc}$ models, respectively. Intuitively, this correlation between the number of CVs and $r_{v}$ (inversely related to the central density) is due to the origination of most CVs in primordial binaries, which are less likely to be disruptive in a less dense cluster (Kremer et al. 2020). The observed number of CVs is consistent with the predicted number from the $r_{v}=2 \mathrm{pc}$ model.

\section{6. $N G C 5897$}

NGC 5897 is a non-core-collapsed cluster at a distance $D \approx 12.6 \mathrm{kpc}$ (Baumgardt et al. 2019b). While the VDP has been measured using both radial velocities (Baumgardt \& Hilker 2018) and proper motions (Baumgardt et al. 2019b), these measurements have not extended into the core of the cluster, and thus do not constrain the dark mass distribution particularly well. We find 14 well-fitting snapshots in the model, N4-RV4-RG8-Z0.01, which differs from the well-fitting model for NGC 288 only in that its metallicity is lower. Thus, between the two clusters, one might expect similarity in their dynamics, but not necessarily in their stellar populations.

\section{7. $N G C 6624$}

One of a handful of clusters with $\gamma$-ray emission $>100 \mathrm{MeV}$ (Tam et al. 2011), NGC 6624 is an interesting, high-metallicity $([\mathrm{M} / \mathrm{H}] \approx-0.44$; Harris 1996, 2010 edition) GC, known to contain at least four MSPs, in addition to two young pulsars (Biggs et al. 1994; Lynch et al. 2012). In the past, it has been argued that the relatively large spin-period derivatives of an MSP near the cluster center is evidence for an IMBH (Peuten et al. 2014; Perera et al. 2017), although these signals have since been found to be consistent with dynamical interactions alone (Gieles et al. 2018; Baumgardt et al. 2019a). Moreover, the cluster is known to contain at least one well-studied LMXB (4U 1820-30; Biggs et al. 1994). Significantly, the cluster lacks any well-fitting snapshots from the unmodified CMC Cluster Catalog - the best-fitting snapshot belongs to the model N8RV0.5-RG2-Z1.0, for which $\tilde{\chi}_{\mathrm{SBP}}^{2}=11.95$. We present NGC 6624 as a test case for introducing new models, in order to fit a known SBP and VDP, not satisfactorily fit by the main $\mathrm{CMC}$ Cluster Catalog.

Without the addition of any other models, the $\mathrm{CMC}$ Cluster Catalog already provides a satisfactory fit for 26 out of 59 of the GCs for which we attempt to locate an analogous model. Of the remaining GCs, there are broadly three reasons why a GC may not be fit well by the $\mathrm{CMC}$ Cluster Catalog. Firstly, the GC's parameters may lie entirely outside of the range of parameter space probed by the CMC Cluster Catalog, although further simulations outside of this parameter space (e.g., new models with larger $N$ than the largest $N$ on the grid) may fit such clusters. Secondly, the GC may lie between points in parameter space sampled by the grid; for example, a GC's initial $r_{v}$ may lie between 1 and 2 pc, but not especially close to either. In this case, the inclusion of models which increase the resolution of the grid would fit these clusters. Finally, the GC may differ from the models in other parameters besides those which have been varied in the grid, e.g., $r_{v}, R_{g}, Z$, and $N$. Such parameters include the initial binary fraction and initial mass function, which are not varied in the CMC Cluster Catalog, but can generally be varied in $\mathrm{CMC}$ to potentially improve GC fits.

NGC 6624 does not have a well-fitting snapshot, according to $s<10$. However, as its closest-fitting snapshots on the main grid do not appear to deviate substantially from the observed data, it is likely to lie within the second aforementioned category: the lack of good fits is probably due to the coarseness of the grid, rather than its limited range. Nevertheless, its closest-fitting snapshots provide guidance as to the manner in which the grid ought to be extended so as to obtain a good fit. Guided by these pre-existing models, we supplement the model grid to better fit NGC 6624, in order to demonstrate targeted cluster fitting with further $\mathrm{CMC}$ simulations.

Notably, $\tilde{\chi}_{\mathrm{SBP}}^{2}=11.95$ for this cluster, indicating that some model SBPs are only slightly discrepant with the observed SBP. Upon inspection, this disagreement arises because the model's results for the SBPs' core brightnesses are higher than observed, while the outer halo brightnesses are slightly lower than observed. Modification of the initial $N$ can narrow these discrepancies (either directly, by altering visible mass, or indirectly, by changing the relaxation time), as can modifying $r_{v}$ (which has been shown to influence progress toward core collapse, see Kremer et al. 2018).

To better fit NGC 6624, we accordingly run six new models, and examine the extent to which they improve (or fail to improve) the fit (Table 2). We consider separately a decrease in initial $N$ to $7 \times 10^{5}$ particles (N7-RV0.5-RG2-Z1.0), and an increase in the virial radius to $r_{v}=0.7 \mathrm{pc}$ (N8-RV0.7-RG2-Z1.0). We also consider an increase in the virial radius to $r_{v}=1.0 \mathrm{pc}$ under three distinct initial particle counts, $N=(6,7,9) \times 10^{5}$, (N6-RV1-RG2-Z1.0, N7-RV1-RG2-Z1.0, and N9-RV1-RG2-Z1.0, respectively). Finally, for $N=7 \times 10^{5}$, we also consider a decrease in metallicity to $Z=0.35 Z_{\odot}$ (N7-RV1-RG2-Z0.35), i.e., the observed metallicity reported by Harris (1996, 2010 edition). As the metallicity is coarsely rounded up to $Z=$ $1.0 Z_{\odot}$ in the main $\mathrm{CMC}$ Cluster Catalog, we consider this latter model, in order to ascertain whether or not the structure of the GC displays fine sensitivity to $Z$.

Of these six models, we find that only N7-RV1-RG2-Z0.35 provides well-fitting snapshots (13, Figures 6 and 7). Within the other models, the best-fitting snapshots from N7-RV0.5-RG2Z1.0, N8-RV0.7-RG2-Z1.0, and N9-RV1.0-RG2-Z1.0 all contain the same central overdensity as the best-fitting snapshots from the CMC Cluster Catalog proper. The models N7-RV1-RG2Z1.0 and N6-RV1-RG2-Z1.0 appear to decrease the core overdensity in the models, although they appear to be slightly overbright at $r_{v} \approx 6^{\prime \prime} \approx 0.2 \mathrm{pc}$.

Of the 13 snapshots that fit NGC 6624 well, all lack MSPs. Most of these snapshots also lack any XRBs, with one snapshot containing a single XRB, and two snapshots containing two XRBs. However, 16 additional snapshots pass the slightly relaxed fitting criterion, $s<15$, with six from different models: N6-RV1-RG2-Z1.0 (with one MSP and two to four XRBs), N7-RV1-RG2-Z1.0 (with two MSPs, and one XRB), and 
Table 2

New Models for NGC 6624

\begin{tabular}{|c|c|c|c|c|c|c|c|c|c|c|}
\hline Model & $N_{\text {snap }}$ & $N_{\text {good }}$ & $\begin{array}{c}r_{v} \\
(\mathrm{pc})\end{array}$ & $\begin{array}{c}R_{g} \\
(\mathrm{kpc})\end{array}$ & {$[\mathrm{M} / \mathrm{H}]$} & $\begin{array}{c}N \\
\left(\times 10^{5}\right)\end{array}$ & $\tilde{\chi}_{\mathrm{SBP}}^{2}$ & $\begin{array}{l}\tilde{\chi}_{\mathrm{VDP}}^{2} \\
\end{array}$ & 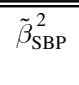 & 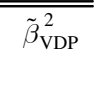 \\
\hline N7-RV0.5-RG2-Z1.0 & 570 & 0 & 0.5 & 2 & 0 & 7 & 14.51 & 2.00 & 6.23 & 0.78 \\
\hline N8-RV0.7-RG2-Z1.0 & 437 & 0 & 0.7 & 2 & 0 & 8 & 12.24 & 3.37 & 9.11 & -2.70 \\
\hline N6-RV1-RG2-Z1.0 & 233 & 0 & 1 & 2 & 0 & 6 & 11.38 & 1.76 & 7.83 & -0.15 \\
\hline N7-RV1-RG2-Z1.0 & 218 & 0 & 1 & 2 & 0 & 7 & 13.60 & 4.05 & 10.94 & -3.90 \\
\hline N9-RV1-RG2-Z1.0 & 317 & 0 & 1 & 2 & 0 & 9 & 21.66 & 13.20 & 21.35 & -13.20 \\
\hline
\end{tabular}

Note. Parameters of additional models generated to better fit NGC 6624, alongside goodness-of-fit measures for closest-fitting snapshots.

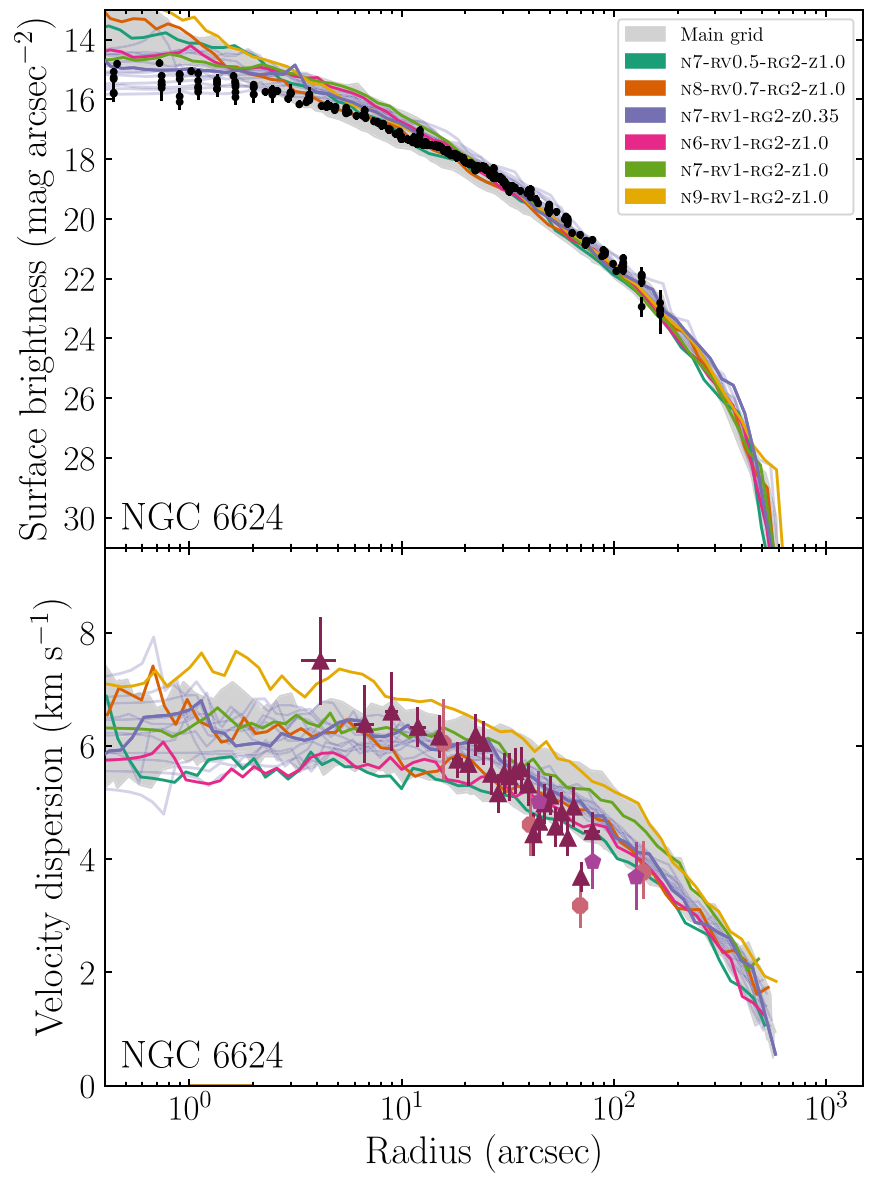

Figure 6. As Figures 2 and 4, but for NGC 6624. As this cluster lacks any wellfitting models from the main CMC Cluster Catalog, we show the values spanned by the 30 best-fitting snapshots from the main CMC Cluster Catalog in the gray region. The best-fitting snapshot from each of the new models is shown, as well as all well-fitting snapshots from the N7-RV1-RG2Z0.35 model.

N8-RV0.7-RG2-Z1.0 (four to five MSPs, and zero to one XRBs). Our models are consistent with NGC 6624's single observed LMXB. Although we find fewer MSPs in our well-fit models, the difference is small, and may be explained by moderate sensitivity of this number to the initial size, compactness, and metallicity of the cluster. Since current observations are not necessarily complete, this could also suggest that our models may somewhat under-produce these types of stellar exotica. However, it is also important to keep in mind that NGC 6624 exists in a more complex high-metallicity regime, and lies behind a moderate amount of extinction. Coupled with uncertainty in terms of the distance from Gaia
( $D=7.19 \pm 0.37 \mathrm{kpc}$, Baumgardt et al. 2019b) and the initial mass function (which may play a significant role; see Weatherford et al. 2021), it remains plausible that both observational and modeling uncertainties could account for the deficit in MSPs and XRBs in the best-fitting models.

\section{Comparison to Cluster Properties}

In recent years, new observational surveys have led to the measurement of a breadth of physical observables across a broad sample of GCs, particularly those in the Milky Way. For each of the seven GCs described in Section 3, we explore model predictions for cluster masses and mass-to-light ratios (Section 4.1), binary fractions (Section 4.2), mass segregation (Section 4.3), and black hole content (Section 4.4), benchmarking these properties to observations whenever available.

\subsection{Cluster Masses and Mass-to-light Ratios}

Although cluster brightness and extent are readily observable quantities, their precise translation to total cluster mass is complex, and generally requires dynamical modeling. Using scaled-up versions of $N$-body simulations, where $N=(1-2) \times$ $10^{5}$, Baumgardt \& Hilker (2018) estimated the total masses and mass-to-light ratios of a number of GCs using radial velocities, including the specific clusters discussed in this paper. They further refined the mass-to-light calculations with additional measurements of $L_{V}$, where they found typical values of $M / L_{V} \sim 1.8$ (Baumgardt et al. 2020). Here, we perform analogous estimates for the total cluster mass, $M$, and massto-light ratio, $M / L$, where $L$ refers to bolometric luminosity (Figure 8).

The seven clusters we examine are estimated to have present-day masses ranging between $\sim 9.2 \times 10^{4} M_{\odot}$ and $\sim$ $1.1 \times 10^{5} M_{\odot}$, and $M / L$ between $\sim 1.5$ and $\sim 1.9$ (Table 3). Our mass estimates are very consistent with those of Baumgardt et al. (2020), except in the case of NGC 5897, where they estimate a significantly larger mass, $M \sim 2 \times 10^{5} M_{\odot}$, and NGC 6624, where they estimate a significantly lower mass $M \sim 7 \times 10^{4} M_{\odot}$. Our mass-to-light ratios are very consistent with those of Baumgardt et al. (2020) in all cases, and are narrowly scattered around $M / L \sim 1.8$.

\subsection{Binary Fraction}

Within GCs, the binary fraction is a photometrically observable property, sensitive to cluster dynamics, particularly in GC cores. The dense environments provided by GCs frequently scatter and eject binary systems, and the dynamical formation of binaries is generally thought to be the halting mechanism for collapse in core-collapsed clusters after the 

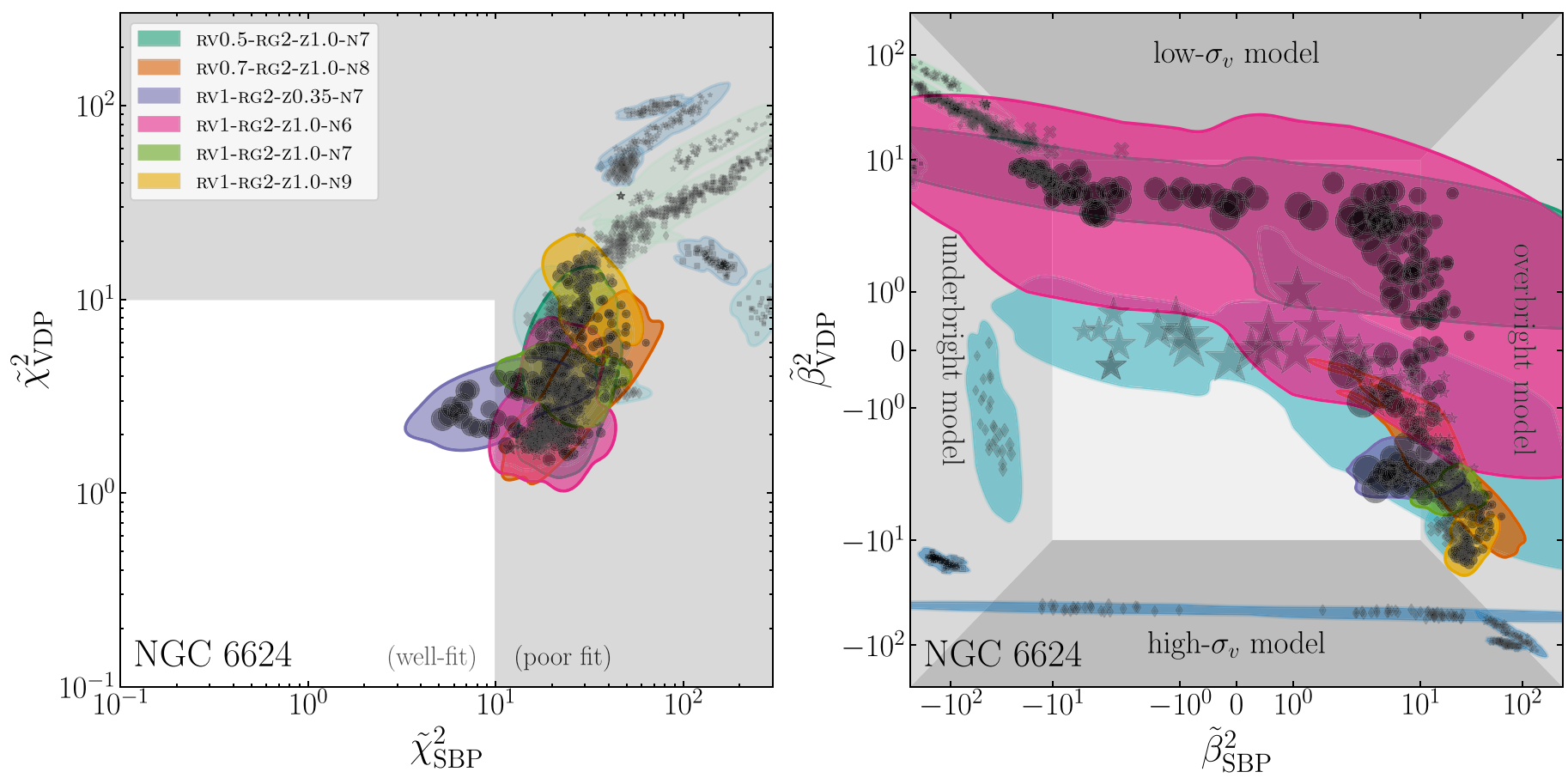

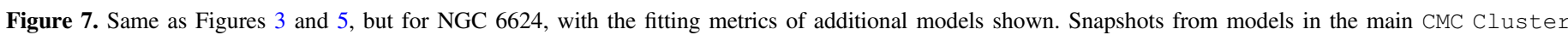
Catalog are shown in washed-out colors for reference.

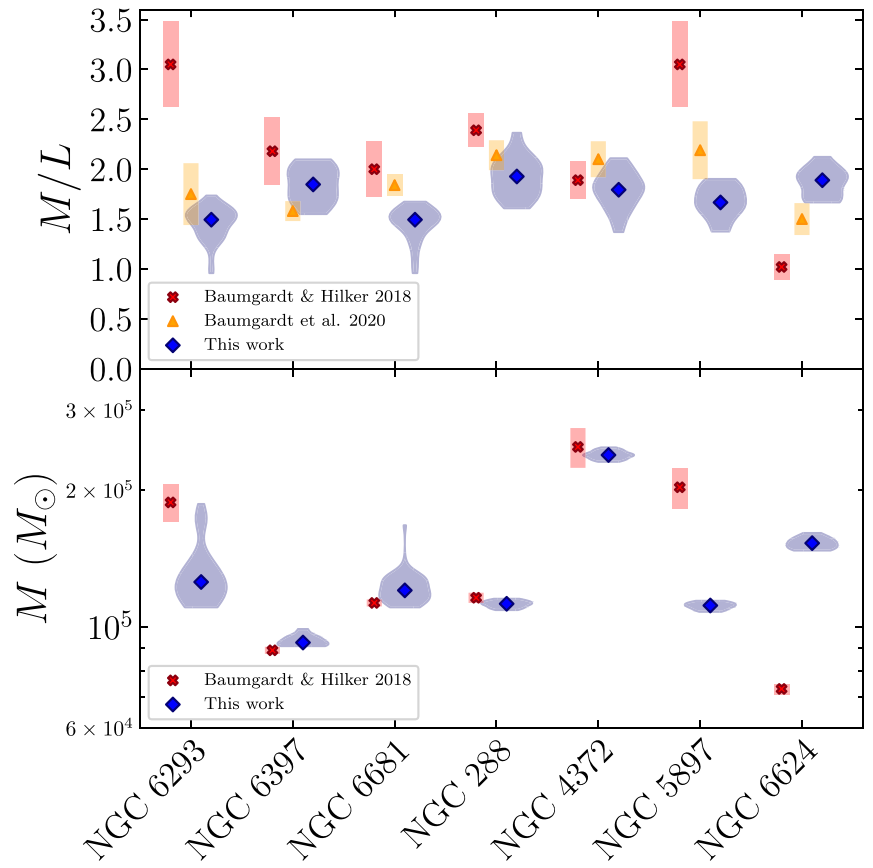

Figure 8. Violin plots of our estimates of the mass-to-light ratios (top), and total cluster masses (bottom), for the core-collapsed clusters, NGC 6293, NGC 6397, NGC 6681, and NGC 6624, and the non-core-collapsed clusters, NGC 288, NGC 4372, and NGC 5897. The widths of the "violins" represent the density of snapshots with a given value of $M / L$ or $M$. Estimates of $M / L$ and $M$ from Baumgardt \& Hilker (2018), using $N$-body simulations combined with scaling relations, and updated estimates of $M / L$ from Baumgardt et al. (2020), using refined measurements of cluster brightnesses, are also shown, where the error bars represent their $1 \sigma$ uncertainties.

expulsion of their black holes (e.g., Chatterjee et al. 2013). Moreover, as binary systems almost always have total fluxes equal to the sum of their component fluxes, main sequence binary systems can be found on the color-magnitude diagram in predictably brighter sequences above the main sequence defined by their mass ratios. In a GC, binaries can either persist from the initial formation of the cluster, or be dynamically generated over time-in the CMC Cluster Catalog, it is assumed in all cases that the primordial binary fraction is $5 \%$, with a flat mass ratio distribution of between $q=0.1$ and 1 . Using the ACS Globular Cluster Survey, Milone et al. (2012) photometrically measured the binary fraction for $59 \mathrm{GCs}$, where the mass ratio $q>0.5,0.6$, and 0.7 individually within three radial regions, $r<r_{c}, r_{c}<r<r_{h}$, and $r>r_{h}$, where $r_{c}$ and $r_{h}$ are as given by Harris (1996, 2010 edition; Figure 9). Of these, five overlap with our seven clusters of interest (they did not report binary fractions for NGC 6293 or NGC 4372).

We calculate the binary fraction, subject to the same minimum mass ratios and radial ranges, for all best-fitting snapshots for each of our seven clusters of interest (Figure 10). To mimic the magnitude cuts applied by Milone et al. (2012), we restrict our sample to a locus on the color-magnitude diagram consistent with binaries whose primary has an F814W magnitude between 0.75 and 3.75 mag below the main sequence turn-off. On the blue edge, we enforce that included sources must have an F606W-F814W color lying no bluer than $0.1 \mathrm{mag}$ of the main sequence turn-off. To calculate a binary fraction for stars above a mass ratio, $q$, we use the SSE main sequence prescription to define a locus on the color-magnitude diagram corresponding to the sum of fluxes due to two main sequence stars with mass ratio $q$. Sources on the red side of the locus are then considered to be binaries. The binary fraction is then calculated by dividing the weight of sources identified as binaries by the total weight of all sources in the magnitude and radial range, with weights defined by Equation (1). Notably, while Milone et al. (2012) additionally applied a general inner radius cut for a number of clusters, of our seven clusters of interest, only NGC 6681 and NGC 6624are affected by such a cut, and, in particular, only the $r_{c}<r<r_{h}$ annulus (they do not 
Table 3

Masses and Mass-to-light Ratios for Seven GCs

\begin{tabular}{|c|c|c|c|c|c|}
\hline \multirow[b]{2}{*}{ Cluster } & \multicolumn{2}{|c|}{ Mass $M\left(M_{\odot}\right)$} & \multicolumn{3}{|c|}{ Mass-to-light Ratio $M / L\left(M_{\odot} / L_{\odot}\right)$} \\
\hline & This work & Baumgardt \& Hilker (2018) & This work & Baumgardt \& Hilker (2018) & Baumgardt et al. (2020) \\
\hline NGC 6293 & $\left(1.26_{-0.15}^{+0.61}\right) \times 10^{5}$ & $(1.88 \pm 0.18) \times 10^{5}$ & $1.49_{-0.53}^{+0.24}$ & $1.67 \pm 0.29$ & $1.75 \pm 0.31$ \\
\hline NGC 6681 & $\left(1.20_{-0.10}^{+0.47}\right) \times 10^{5}$ & $(1.13 \pm 0.02) \times 10^{5}$ & $1.49_{-0.53}^{+0.18}$ & $2.00 \pm 0.28$ & $1.84 \pm 0.11$ \\
\hline NGC 288 & $\left(1.13_{-0.03}^{+0.03}\right) \times 10^{5}$ & $(1.16 \pm 0.03) \times 10^{5}$ & $1.93_{-0.32}^{+0.44}$ & $2.39 \pm 0.17$ & $2.14 \pm 0.15$ \\
\hline NGC 4372 & $\left(2.39_{-0.08}^{+0.09}\right) \times 10^{5}$ & $(2.49 \pm 0.25) \times 10^{5}$ & $1.79_{-0.42}^{+0.31}$ & $1.89 \pm 0.19$ & $2.10 \pm 0.18$ \\
\hline
\end{tabular}

Note. Cluster mass and mass-to-light ratio for NGC 6293, NGC 6397, NGC 6681, NGC 288, NGC 4372, NGC 5897, and NGC 6624. The uncertainty bars reported here are taken to span the entire range of values for $M$ and $M / L$ appearing in well-fitting snapshots for a given cluster. Values of $M$ from Baumgardt \& Hilker (2018), as well as $M / L$ from Baumgardt \& Hilker (2018), and Baumgardt et al. (2020) for these clusters are also reproduced above.

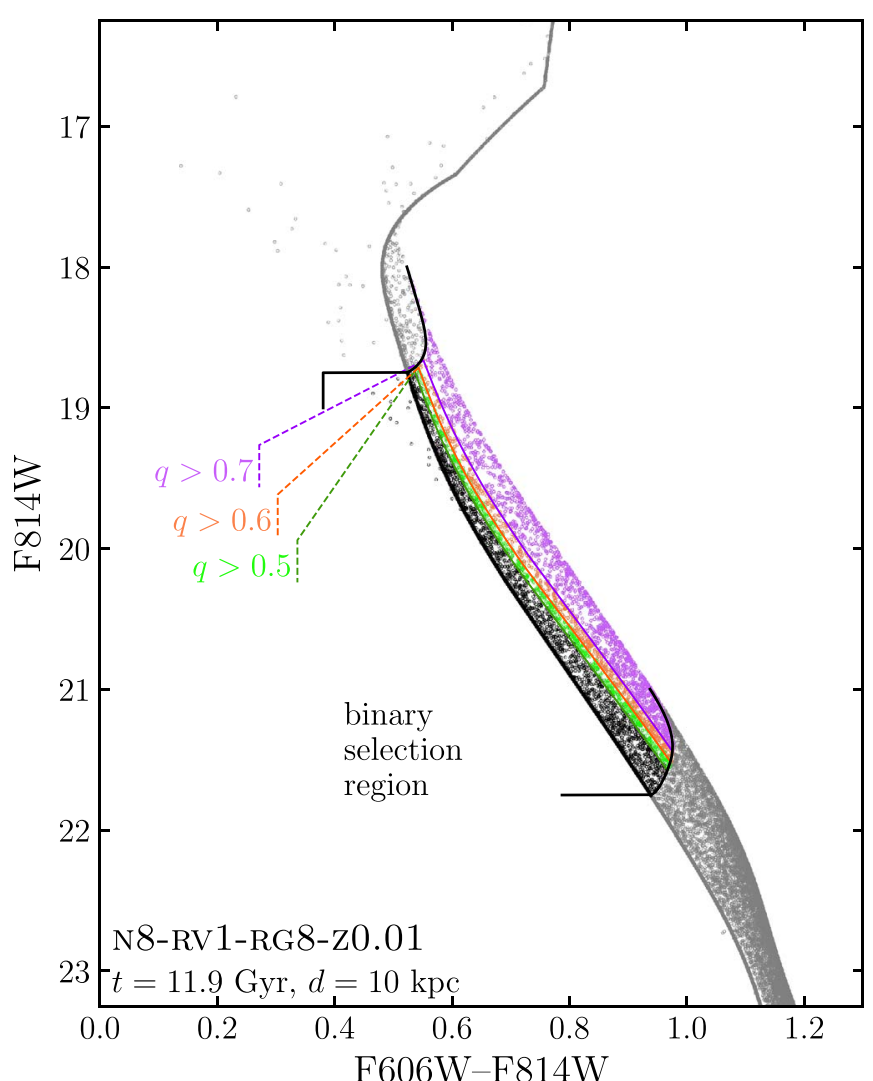

Figure 9. Synthetic color-magnitude diagram, showing the cuts applied to the simulated catalog to find binaries where $q>0.5, q>0.6$, and $q>0.7$. We apply cuts to mimic the observational procedure of Milone et al. (2012; ACS Globular Cluster Survey) as closely as possible.

report binary fractions for $r<r_{c}$ ). However, in order to keep applied cuts relatively consistent between the clusters, we omit this cut for these particular GCs.

We find reasonable consistency between the model binary fractions and the data in most cases. One notable exception is the binary fraction within the inner spatial bins of NGC 6397, where our models appear to predict binary fractions of up to $\sim 3$ times the observed value-even here, the data and models come back into agreement in the outermost bin. Another is in the $r_{c}<r<r_{h}$ bin of NGC 6624, where Milone et al. (2012) reported very low binary fractions, curiously lower than the binary fraction of the outskirts of the cluster $\left(r>r_{h}\right)$. However,

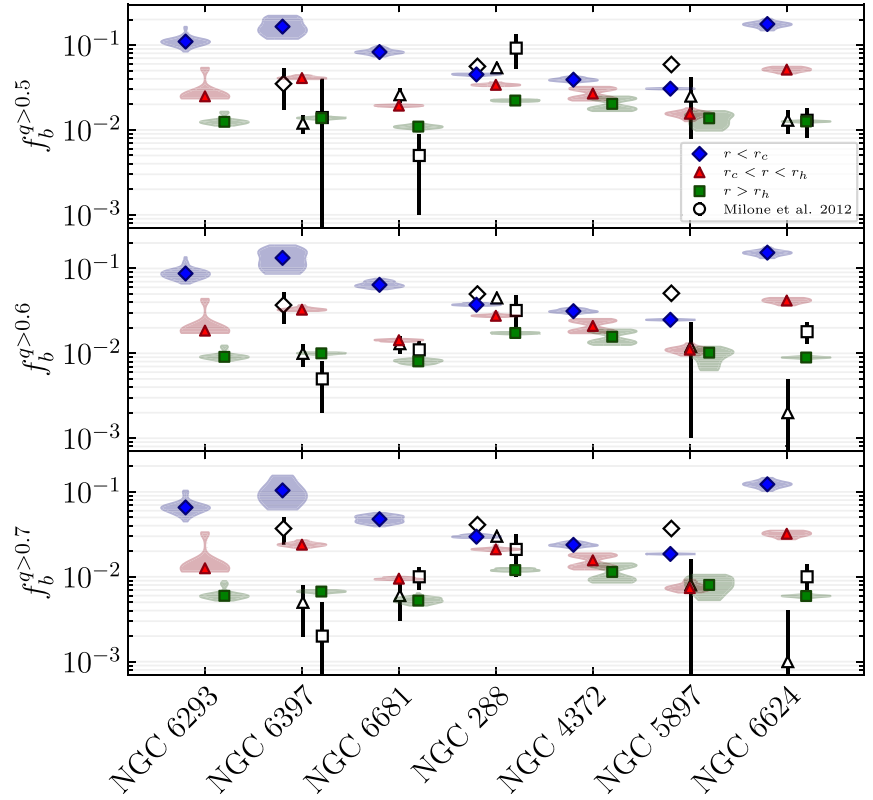

Figure 10. Binary fraction of seven GCs with mass fractions $q>0.5$ (top), $q>0.6$ (center), and $q>0.7$ (bottom), where $r<r_{c}$ (blue), $r_{c}<r<r_{h}$ (red), and $r>r_{h}$ (green). For the model values, the horizontal width of the violin plot point refers to the density of well-fitting snapshots with that particular binary fraction value. Observed values given by Milone et al. (2012; ACS Globular Cluster Survey) are also shown.

in general, measurement of the binary fraction within corecollapsed clusters is relatively difficult for a number of reasons. Firstly, formally, core-collapsed clusters do not have a welldefined core radius, and observational definitions of the core radius tend to be quite small (e.g., $r_{c} \sim 0.03 \mathrm{pc}$ for NGC 6397), with conversions from angular units to physical distances being very sensitive to heliocentric distance. As binaries are dynamically generated and then burned in large numbers in the cores of core-collapsed clusters, the binary fraction is expected to vary substantially with respect to distance from the GC center. Moreover, in the central regions of such clusters, stellar density is extremely large, implying relatively low completeness.

Overall, we note acceptable agreement between our predictions and observations for non-core-collapsed clusters, as well as the outer regions of core-collapsed clusters. This provides a degree of reassurance that $\mathrm{CMC}$ can sensibly replicate the binary 


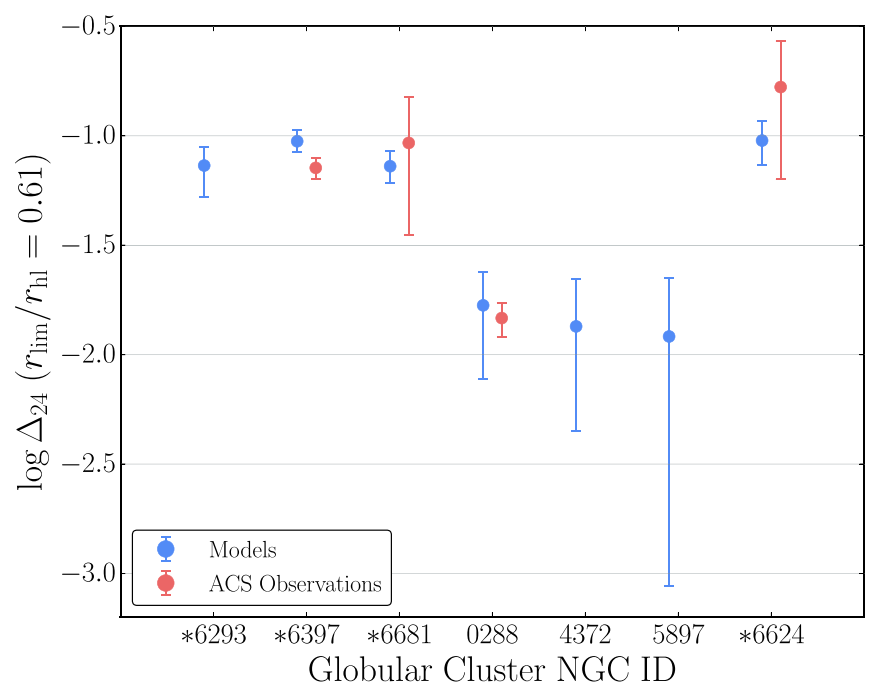

Figure 11. Mass segregation metric, $\Delta_{24}$, calculated for both well-fitting snapshots to our seven GCs of interest, and also directly from the ACS Globular Cluster Survey data, where available. In both cases, the plotted uncertainties correspond to a $95 \%$ confidence interval, with the uncertainty in the model values taking into account variations due to different twodimensional projections, as estimated by ten different realizations per snapshot. General agreement between the simulated and observed values is apparent.

populations of realistic GCs, although the fixed assumed binary fraction of $5 \%$ in a flat mass ratio distribution, combined with a complex initial to final binary fraction mapping, complicates the picture somewhat.

\subsection{Mass Segregation}

Over thermodynamically long timescales, massive stars are expected to sink to the center of the cluster through dynamical friction. It can be shown that a population of stars with mass $m$ will segregate within a cluster on a timescale $t_{m, \mathrm{MS}} \sim(\langle m\rangle / m) t_{\mathrm{rlx}}$, where $\langle m\rangle$ is the mean mass of the cluster (Portegies Zwart et al. 2010). As $t_{\mathrm{rlx}} \sim$ few $\times 10^{9} \mathrm{yr}$ for typical GCs, mass segregation in GCs is readily identified as a preferential clustering of massive stars closer to the center of the cluster. Moreover, given that "dark" objects, such as black holes, and other stellar remnants also participate in mass segregation, they may influence the observed metrics of mass segregation in nontrivial ways.

Here, we examine the capacity of the SBP and VDP alone to predict the degree of mass segregation within a cluster. In particular, in accordance with Weatherford et al. (2020), we define Population II stars as the "high-mass" population, where $L_{\mathrm{MSTO}} / 5<L<L_{\mathrm{MSTO}}$, and Population IV stars as the "lowmass" population, such that $L_{\mathrm{MSTO}} / 125<L<L_{\mathrm{MSTO}} / 25$, and where $L_{\mathrm{MSTO}}$ is the luminosity of the main sequence turn-off. We then parameterize the mass segregation for each cluster, $\Delta_{24}$, defined as the difference between the median projected radial distance of Population II stars and Population IV stars, normalized by the half-light radius of the cluster.

Note that $\Delta_{24}$ is straightforwardly calculated both for simulations and observed clusters. In particular, using the ACS Globular Cluster Survey, we reproduce this calculation for four of our seven clusters of interest with suitable observations, following the procedure of Weatherford et al. (2018, 2020). We take into account incompleteness in the observed catalogs, estimated from artificial star tests. We then compare these to the distribution of $\Delta_{24}$ within well-fitting snapshots for our seven clusters (Figure 11). Within the simulated data, we mimic the limited field of view of the data by restricting it to stars within a projected radius of $61 \%$ of the half-light radius (which is the highest value of the four clusters that can be accommodated by the data). In cases where comparison is possible, we find very strong agreement between the simulated and observed $\Delta_{24}$ except in NGC 6397, where the simulations slightly overestimate the degree of mass segregation.

\subsection{Black Holes}

While black holes (BHs) in GCs are very difficult to detect directly, their presence and number can be indirectly inferred by examining their effect on a GC's dynamical state. For example, by considering a grid of CMC models finely gridded over initial virial radius, Kremer et al. (2019) demonstrated the importance of BHs in the halting of core collapse in GCs NGC 3201, NGC 6656 (M22), and NGC 6254 (M10), and noted their likely absence in NGC 6752, which is core-collapsed. These $\mathrm{BH}$ populations are, in turn, intimately related to the cluster's initial size. Along a similar vein, trends in the core radius with age for massive clusters in Milky Way satellite galaxies in the seminal Mackey \& Gilmore catalogs (Mackey \& Gilmore 2003a, 2003b, 2003c) have been interpreted as evidence for the role of BHs in the clusters' bulk evolution (see, e.g., Mackey et al. 2008, which reproduces these observed trends in $N \sim 10^{5}$-body simulations).

Evidence of the effect of $\mathrm{BH}$ populations on the structure of a $\mathrm{GC}$ is also apparent in the spatial distribution of stars in different mass ranges. In particular, using CMC models, Weatherford et al. (2020) constrained the number of BHs in GCs by taking advantage of an anticorrelation between the extent of mass segregation in a cluster and its $\mathrm{BH}$ population, a trend quantifiable in $\mathrm{CMC}$ models. Intuitively, this anticorrelation arises from the rapid segregation of a GC's black hole population, followed by dynamical heating of the massive star population, and their typical distances from the cluster center.

For a similar reason, the presence of a $\mathrm{BH}$ population in a GC halts core collapse-large $\mathrm{BH}$ populations transfer significant energy to their host cluster's stellar population through binary-mediated dynamics, preventing core collapse of the bulk stellar population (e.g., Kremer et al. 2020). This manifests in terms of a flattened core surface brightness, as well as a heightened dynamical temperature in the core of the cluster. This motivates the use of observed SBPs and VDPs to constrain the $\mathrm{BH}$ populations of GCs, which can in turn be accomplished by analyzing the simulated stellar populations of their best-fitting $\mathrm{CMC}$ models.

For each of the seven GCs of interest, we calculate the median, 18th $(-1 \sigma)$ and 84th $(+1 \sigma)$ percentiles, and minimum and maximum number of $\mathrm{BH}$ in well-fitting snapshots (Table 4). As expected, all three of the core-collapse clusters examined have fully single-digit BH counts. Of their sample of 50 GCs, four of the GCs for which Weatherford et al. (2020) have estimated BH counts coincide with our seven: NGC 6397, NGC 6681, NGC 288, and NGC 6624. Reassuringly, our BH predictions are consistent with theirs when the metric parameterizing mass segregation is consistent with the definition in Section 4.3. Moreover, both Weatherford et al. (2020) and our work broadly reflect the tendency of core-collapsed clusters to have fewer BHs, reinforcing the scenario whereby black holes provide the dominant mechanism for halted core 
Table 4

Predicted Black Hole Counts for Seven GCs

\begin{tabular}{|c|c|c|c|c|c|c|c|c|c|c|}
\hline \multirow[b]{2}{*}{ Cluster } & \multicolumn{5}{|c|}{ This work } & \multicolumn{5}{|c|}{ Weatherford et al. (2020) } \\
\hline & Min. & $-1 \sigma$ & Median & $+1 \sigma$ & Max. & $-2 \sigma$ & $-1 \sigma$ & Median & $+1 \sigma$ & $+2 \sigma$ \\
\hline NGC $6293^{c}$ & 0.00 & 0.00 & 0.00 & 0.00 & 16.00 & $\ldots$ & $\ldots$ & $\ldots$ & $\ldots$ & $\ldots$ \\
\hline NGC $6397^{c}$ & 1.00 & 1.00 & 1.00 & 1.00 & 1.00 & 0.00 & 0.00 & 0.61 & 1.80 & 4.06 \\
\hline NGC $6681^{c}$ & 0.00 & 0.00 & 0.00 & 0.00 & 7.00 & 0.00 & 1.21 & 5.02 & 10.10 & 16.30 \\
\hline NGC 288 & 48.00 & 54.40 & 70.00 & 79.20 & 88.00 & 2.24 & 9.93 & 18.2 & 26.6 & 46.9 \\
\hline NGC 4372 & 93.00 & 106.44 & 217.00 & 347.68 & 375.00 & $\ldots$ & $\ldots$ & $\ldots$ & $\ldots$ & $\ldots$ \\
\hline NGC 5897 & 44.00 & 44.24 & 56.00 & 64.76 & 67.00 & $\ldots$ & $\ldots$ & $\ldots$ & $\ldots$ & $\ldots$ \\
\hline $\mathrm{NGC} 6624^{c}$ & 2.00 & 2.00 & 6.00 & 6.00 & 9.00 & 0.70 & 19.60 & 23.20 & 26.80 & 31.1 \\
\hline
\end{tabular}

Note. The number of BHs in well-fitting snapshots for seven Milky Way GCs. For each cluster, the median, maximum, and minimum, 16th and 84th percentiles are reported. Core-collapsed clusters are identified using the subscript, $c$. For reference, we have also included the number of black holes as estimated by Weatherford et al. (2020) using the observed mass segregation.

collapse in the majority of GCs today. Although both this work and that of Weatherford et al. (2020) calibrate $N_{\mathrm{BH}}$ to the same grid of models, we obtain constraints from two distinct observables (the SBP and VDP versus the degree of mass segregation in the cluster). This indicates at least concordance with the idea that both the (suppressed) degree of mass segregation and cluster dynamics are broadly driven by a single $\mathrm{BH}$ population at the center of a GC (or the lack thereof). Nevertheless, given a lack of direct observations of $N_{\mathrm{BH}}$, the actual size of this population remains highly uncertain.

\section{Conclusion}

The approach to GC modeling enabled by $\mathrm{CMC}$ provides a balanced approach to running accurate, long-timescale simulations of realistically large GCs in practical runtimes, without reliance on scaling relations to deduce cluster parameters. This opens the door to holistic, direct comparisons of observations to extensive model grids over realistic GC parameter spaces. Accordingly, we have presented a scheme for identifying wellfitting simulation snapshots from the CMC Cluster Catalog. Out of 59 Milky Way GCs, we find that the $\mathrm{CMC}$ Cluster Catalog provides good fits to $26 \mathrm{GCs}$ as is. As illustrative examples, we focused specifically on six of these well-fit clusters in our database. In order to demonstrate that the number of good fits can readily be extended with the addition of new CMC models, we have detailed a procedure for augmenting the model grid to fit a seventh GC, NGC 6624, which is not well fit by any snapshot in the original $\mathrm{CMC}$ cluster Catalog. We have examined the clusters' predicted masses, mass-to-light ratios, binary fractions, and black hole counts, finding reasonable consistency with previous works and observations in most cases. The predicted numbers of of cataclysmic variables, low-mass X-ray binaries, and millisecond pulsars have also been reported, where analogous observations exist, with consistency found in all cases, with the possible exception of NGC 6624.

Motivated by the desire to extend the utility of this method to a wider range of clusters, as well as by the precision of the comparison, we suggest a number of potential refinements to this procedure: (1) extension of the grid both to parameters within the current parameter range (to increase the parameter space grid resolution), and outside (to extend the grid to fit those GCs not represented in the current model grid), (2) variation of additional parameters, such as the binarity or initial mass function, in order to better capture the full diversity of possible GC evolution histories, (3) including the observed mass function slope in constraints on the GC as a further axis of comparison (such observations are already available for a number of Milky Way GCs, e.g., Sollima \& Baumgardt 2017), and (4) proactively leveraging observed stellar counts for populations such as CVs, XRBs, and blue stragglers as additional constraints in matching models.

We make available a set of functions for analyzing GC models generated using $\mathrm{CMC}$, including the already publicly available CMC Cluster Catalog, ${ }^{8}$ as well as files containing the model SBPs, VDPs, and other parameters for the snapshots considered in this work.

We thank L. Clifton Johnson for invaluable discussions and advice, and the anonymous referee for their useful suggestions. This work was supported by NSF grant AST-1716762, and by the computational resources and staff contributions provided for the Quest high-performance computing facility at Northwestern University. N.Z.R. acknowledges support from the Illinois Space Grant Consortium, and the Dominic Orr Graduate Fellowship. K.K. is supported by an NSF Astronomy and Astrophysics Postdoctoral Fellowship under award No. AST-2001751. N.C.W. acknowledges support from the CIERA Riedel Graduate Fellowship at Northwestern University, as well as the NSF GK-12 Fellowship Program under grant No. DGE-0948017. S.C. acknowledges support from the Department of Atomic Energy, Government of India, under project no. 12-R\&D-TFR-5.02-0200. This research has made use of the SVO Filter Profile Service (http://svo2.cab.inta-csic.es/theory/fps/) supported from the Spanish MINECO through grant AYA2017-84089.

Software: Astropy (The Astropy Collaboration 2013), IPython (Pérez \& Granger 2007), Matplotlib (Hunter 2007), NumPy (Oliphant 2006; Harris et al. 2020), SciPy (Jones et al. 2006; Virtanen et al. 2020), Pandas (McKinney 2010), Cluster Monte Carlo (Pattabiraman \& Umbreit 2013), cmctoolkit (Rui et al. 2021), COSMIC (Breivik et al. 2020), FEWBODY (Fregeau et al. 2004).

\section{Appendix \\ Best Fits to 59 Observed GCs}

In this appendix, we include a table of GCs with available SBPs and VDPs, such that both have more than five data points. For GCs for which at least one snapshot where $s=$ $\max \left(\tilde{\chi}_{\mathrm{SBP}}^{2}, \tilde{\chi}_{\mathrm{VDP}}^{2}\right)<10$, we report in Table 5 all model parameters with well-fitting snapshots, together with the number

\footnotetext{
https://cmc.ciera.northwestern.edu/home/
} 
Table 5

Best-fitting Model Parameters, Fitting Figures of Merit, and Estimated Black Hole Populations for Milky Way GCs

\begin{tabular}{|c|c|c|c|c|c|c|c|c|c|c|c|c|}
\hline Name & $N_{\mathrm{SBP}}$ & $N_{\mathrm{VDP}}$ & $\begin{array}{c}r_{v} \\
(\mathrm{pc})\end{array}$ & $\begin{array}{c}r_{g} \\
(\mathrm{kpc})\end{array}$ & {$[\mathrm{M} / \mathrm{H}]$} & $\begin{array}{c}N \\
\left(\times 10^{5}\right)\end{array}$ & $N_{\text {good }}$ & $s$ & $\tilde{\chi}_{\mathrm{SBP}}^{2}$ & $\tilde{\chi}_{\mathrm{VDP}}^{2}$ & $\tilde{\beta}_{\mathrm{SBP}}^{2}$ & $\tilde{\beta}_{\mathrm{VDP}}^{2}$ \\
\hline \multirow[t]{2}{*}{ NGC 6553} & 105 & 9 & 2.0 & 2 & 0 & 16 & 73 & 0.92 & 0.92 & 0.91 & -0.13 & -0.70 \\
\hline & & & 2.0 & 2 & 0 & 8 & 3 & 9.02 & 7.01 & 9.02 & -6.97 & 9.02 \\
\hline \multirow[t]{2}{*}{ NGC 4372} & 23 & 12 & 4.0 & 8 & -2 & 8 & 30 & 1.06 & 1.06 & 0.53 & 0.34 & -0.03 \\
\hline & & & 2.0 & 8 & -2 & 8 & 30 & 5.34 & 5.34 & 0.95 & 5.34 & -0.63 \\
\hline NGC 6352 & 56 & 6 & 2.0 & 2 & -1 & 4 & 11 & 1.56 & 1.56 & 0.57 & 1.06 & 0.57 \\
\hline NGC 288 & 92 & 40 & 4.0 & 8 & -1 & 4 & 16 & 1.88 & 1.23 & 1.88 & 0.70 & 1.79 \\
\hline NGC 6723 & 189 & 13 & 2.0 & 2 & -1 & 8 & 29 & 1.88 & 1.88 & 0.82 & -1.10 & 0.40 \\
\hline \multirow[t]{2}{*}{ NGC 6569} & 142 & 5 & 1.0 & 2 & -1 & 16 & 64 & 2.34 & 1.30 & 2.34 & 0.26 & -1.76 \\
\hline & & & 1.0 & 2 & -1 & 8 & 30 & 3.62 & 1.90 & 3.62 & -0.63 & 3.62 \\
\hline NGC 6656 & 146 & 53 & 1.0 & 2 & -2 & 16 & 70 & 3.17 & 1.74 & 3.17 & 1.00 & 2.78 \\
\hline NGC 5897 & 90 & 7 & 4.0 & 8 & -2 & 4 & 14 & 3.84 & 1.46 & 3.84 & 0.02 & 3.84 \\
\hline \multirow{3}{*}{ NGC 6779} & 152 & 6 & 1.0 & 8 & -2 & 8 & 16 & 3.68 & 3.68 & 2.28 & 3.16 & -1.91 \\
\hline & & & 2.0 & 8 & -2 & 8 & 14 & 4.04 & 4.04 & 1.26 & -0.06 & -0.79 \\
\hline & & & 2.0 & 8 & -2 & 4 & 7 & 4.70 & 2.32 & 4.70 & -1.68 & 4.70 \\
\hline NGC 1904 & 355 & 11 & 1.0 & 20 & -2 & 8 & 7 & 4.21 & 4.21 & 2.92 & 2.68 & -2.92 \\
\hline NGC 5986 & 85 & 8 & 1.0 & 2 & -2 & 16 & 20 & 4.36 & 4.36 & 0.91 & -0.94 & 0.13 \\
\hline NGC 6681 & 150 & 40 & 0.5 & 2 & -2 & 8 & 49 & 4.37 & 3.71 & 4.37 & 3.60 & 4.27 \\
\hline \multirow{2}{*}{ NGC 6541} & 128 & 13 & 2.0 & 2 & -2 & 16 & 36 & 4.38 & 3.11 & 4.38 & -1.18 & 1.69 \\
\hline & & & 1.0 & 2 & -2 & 16 & 76 & 6.91 & 1.79 & 6.91 & 0.75 & -1.91 \\
\hline \multirow[t]{2}{*}{ NGC 5024} & 223 & 8 & 2.0 & 20 & -2 & 16 & 75 & 5.02 & 5.02 & 2.14 & -3.41 & -1.19 \\
\hline & & & 1.0 & 20 & -2 & 16 & 3 & 9.23 & 9.23 & 2.46 & 0.76 & -1.50 \\
\hline \multirow[t]{2}{*}{ NGC 6293} & 234 & 7 & 1.0 & 2 & -2 & 8 & 8 & 5.06 & 5.06 & 1.35 & -1.21 & 1.04 \\
\hline & & & 0.5 & 2 & -2 & 8 & 51 & 5.26 & 5.26 & 1.92 & 2.29 & 1.89 \\
\hline \multirow[t]{2}{*}{ NGC 6712} & 58 & 8 & 1.0 & 2 & -1 & 8 & 30 & 5.97 & 5.97 & 3.79 & -0.58 & -3.79 \\
\hline & & & 2.0 & 2 & -1 & 8 & 17 & 6.06 & 6.06 & 0.39 & -5.26 & -0.36 \\
\hline NGC 6397 & 344 & 50 & 1.0 & 8 & -2 & 4 & 11 & 6.16 & 6.16 & 4.78 & 4.24 & 3.87 \\
\hline \multirow[t]{2}{*}{ NGC 3201} & 84 & 32 & 2.0 & 8 & -2 & 4 & 8 & 6.17 & 1.51 & 6.17 & 0.90 & 5.99 \\
\hline & & & 2.0 & 8 & -2 & 8 & 7 & 8.64 & 8.24 & 8.64 & 7.11 & -8.60 \\
\hline \multirow[t]{2}{*}{ NGC 6539} & 94 & 5 & 1.0 & 2 & -1 & 16 & 64 & 3.99 & 3.38 & 3.99 & -2.41 & -3.99 \\
\hline & & & 1.0 & 2 & -1 & 8 & 13 & 6.32 & 6.32 & 0.59 & -3.49 & 0.59 \\
\hline NGC 6121 & 230 & 26 & 1.0 & 8 & -1 & 4 & 16 & 6.86 & 4.09 & 6.86 & 2.63 & 5.85 \\
\hline \multirow[t]{3}{*}{ NGC 1261} & 129 & 10 & 2.0 & 20 & -1 & 8 & 12 & 7.05 & 7.05 & 1.10 & -0.37 & -0.86 \\
\hline & & & 1.0 & 20 & -1 & 8 & 1 & 8.89 & 8.89 & 2.12 & 7.48 & -1.71 \\
\hline & & & 2.0 & 20 & -1 & 4 & 1 & 9.70 & 9.70 & 5.12 & -9.57 & 5.12 \\
\hline NGC 1851 & 102 & 42 & 0.5 & 20 & -1 & 16 & 8 & 7.66 & 2.08 & 7.66 & -1.57 & -6.31 \\
\hline NGC 6496 & 30 & 9 & 4.0 & 2 & 0 & 8 & 12 & 7.83 & 5.65 & 7.83 & 5.65 & -5.00 \\
\hline Ter 5 & 62 & 6 & 1.0 & 2 & 0 & 16 & 44 & 8.43 & 2.65 & 8.43 & 0.91 & 8.43 \\
\hline NGC 5286 & 98 & 10 & 1.0 & 8 & -2 & 16 & 3 & 8.91 & 8.91 & 0.49 & -0.76 & 0.29 \\
\hline & & & 1.0 & 8 & -2 & 8 & 10 & 9.93 & 2.23 & 9.93 & -1.04 & 9.92 \\
\hline NGC 6171 & 102 & 18 & 2.0 & 2 & -1 & 8 & 1 & 9.32 & 9.32 & 4.44 & -2.90 & -4.44 \\
\hline NGC 6304 & 108 & 6 & 2.0 & 2 & 0 & 8 & 0 & 10.68 & 10.68 & 1.40 & -4.16 & -1.32 \\
\hline NGC 6205 & 128 & 14 & 1.0 & 8 & -2 & 16 & 0 & 11.43 & 11.43 & 1.95 & 10.39 & 1.28 \\
\hline NGC 6809 & 115 & 13 & 2.0 & 2 & -2 & 8 & 0 & 11.77 & 11.77 & 2.69 & 9.15 & 0.83 \\
\hline NGC 6624 & 279 & 33 & 0.5 & 2 & 0 & 8 & 0 & 11.95 & 11.95 & 1.71 & -2.65 & -0.28 \\
\hline NGC 6218 & 144 & 12 & 2.0 & 2 & -1 & 8 & 0 & 12.07 & 12.07 & 5.05 & -6.16 & -5.05 \\
\hline NGC 362 & 241 & 53 & 0.5 & 8 & -1 & 16 & 0 & 12.15 & 1.94 & 12.15 & -0.12 & -10.79 \\
\hline NGC 5272 & 91 & 21 & 1.0 & 8 & -2 & 16 & 0 & 12.42 & 12.42 & 1.68 & 10.71 & -0.52 \\
\hline NGC 6366 & 28 & 9 & 2.0 & 2 & -1 & 4 & 0 & 13.75 & 13.75 & 1.24 & 12.54 & -1.18 \\
\hline NGC 4590 & 240 & 7 & 2.0 & 8 & -2 & 8 & 0 & 13.96 & 13.96 & 8.47 & 12.98 & -8.47 \\
\hline NGC 6626 & 326 & 11 & 0.5 & 2 & -1 & 16 & 0 & 14.27 & 14.27 & 0.90 & 14.08 & -0.42 \\
\hline NGC 6402 & 84 & 11 & 1.0 & 2 & -1 & 16 & 0 & 14.31 & 14.31 & 12.31 & -13.00 & 12.31 \\
\hline NGC 6362 & 58 & 31 & 2.0 & 8 & -1 & 4 & 0 & 14.74 & 14.74 & 1.34 & 9.28 & 0.48 \\
\hline NGC 7089 & 269 & 21 & 1.0 & 8 & -2 & 16 & 0 & 15.41 & 8.84 & 15.41 & -6.97 & 15.41 \\
\hline NGC 6273 & 125 & 9 & 1.0 & 2 & -2 & 16 & 0 & 15.66 & 9.76 & 15.66 & -8.64 & 15.66 \\
\hline NGC 6522 & 274 & 11 & 0.5 & 2 & -1 & 16 & 0 & 18.60 & 18.60 & 1.75 & 16.70 & -1.68 \\
\hline NGC 7099 & 297 & 22 & 1.0 & 8 & -2 & 4 & 0 & 18.68 & 9.98 & 18.68 & 5.20 & 18.53 \\
\hline NGC 6752 & 334 & 50 & 1.0 & 8 & -2 & 8 & 0 & 19.90 & 5.12 & 19.90 & -0.16 & 19.72 \\
\hline NGC 5904 & 125 & 52 & 1.0 & 8 & -1 & 16 & 0 & 23.14 & 23.14 & 6.81 & -21.65 & -6.45 \\
\hline NGC 5927 & 57 & 40 & 2.0 & 2 & 0 & 8 & 0 & 24.27 & 20.75 & 24.27 & -16.31 & 24.25 \\
\hline NGC 6093 & 267 & 11 & 1.0 & 2 & -2 & 16 & 0 & 26.16 & 26.16 & 11.22 & 6.34 & 11.19 \\
\hline NGC 7078 & 405 & 50 & 1.0 & 8 & -2 & 16 & 0 & 29.27 & 20.50 & 29.27 & -18.84 & 28.64 \\
\hline NGC 6535 & 58 & 8 & 4.0 & 2 & -2 & 16 & 0 & 30.08 & 15.36 & 30.08 & 15.18 & -30.08 \\
\hline NGC 5824 & 81 & 5 & 1.0 & 20 & -2 & 32 & 0 & 32.22 & 32.22 & 0.53 & 0.46 & 0.53 \\
\hline
\end{tabular}


Table 5

(Continued)

\begin{tabular}{|c|c|c|c|c|c|c|c|c|c|c|c|c|}
\hline Name & $N_{\mathrm{SBP}}$ & $N_{\mathrm{VDP}}$ & $\begin{array}{c}r_{v} \\
(\mathrm{pc})\end{array}$ & $\begin{array}{c}r_{g} \\
(\mathrm{kpc})\end{array}$ & {$[\mathrm{M} / \mathrm{H}]$} & $\begin{array}{c}N \\
\left(\times 10^{5}\right)\end{array}$ & $N_{\text {good }}$ & $s$ & $\tilde{\chi}_{\mathrm{SBP}}^{2}$ & $\tilde{\chi}_{\mathrm{VDP}}^{2}$ & $\tilde{\beta}_{\mathrm{SBP}}^{2}$ & $\tilde{\beta}_{\mathrm{VDP}}^{2}$ \\
\hline NGC 6254 & 161 & 23 & 1.0 & 2 & -2 & 8 & 0 & 33.53 & 33.53 & 2.18 & 26.58 & 0.24 \\
\hline NGC 6341 & 99 & 39 & 1.0 & 8 & -2 & 16 & 0 & 58.86 & 58.86 & 5.23 & -57.97 & -5.19 \\
\hline NGC 2419 & 139 & 5 & 2.0 & 20 & -2 & 32 & 0 & 119.26 & 119.26 & 3.05 & 26.26 & -3.05 \\
\hline NGC 2808 & 304 & 48 & 0.5 & 8 & -1 & 16 & 0 & 167.77 & 30.86 & 167.77 & -30.15 & 167.77 \\
\hline NGC 6266 & 227 & 42 & 0.5 & 2 & -1 & 16 & 0 & 199.06 & 17.86 & 199.06 & -17.84 & 199.06 \\
\hline NGC 6441 & 158 & 37 & 1.0 & 2 & 0 & 16 & 0 & 238.98 & 187.23 & 238.98 & -187.23 & 238.98 \\
\hline NGC 5139 & 73 & 65 & 1.0 & 8 & -2 & 16 & 0 & 1086.16 & 151.20 & 1086.16 & -151.20 & 1086.16 \\
\hline
\end{tabular}

Note. For 59 Milky Way GCs, we report the number $N_{\mathrm{SBP}}$ of data points in the SBP, number $N_{\mathrm{VDP}}$ of data points in the VDP, initial virial radius, galactocentric distance, metallicity, and initial particle number of the well-fitting or best-fitting model(s) (depending on whether the GC is well fit), the number of well-fitting snapshots, $N_{\text {good }}, s, \tilde{\chi}_{\mathrm{SBP}}^{2}, \tilde{\chi}_{\mathrm{VDP}}^{2}, \tilde{\beta}_{\mathrm{SBP}}^{2}$, and $\tilde{\beta}_{\mathrm{VDP}}^{2}$.

of well-fitting snapshots, $N_{\text {good }}$, and the fitting and diagnostic parameters, $s, \tilde{\chi}_{\mathrm{SBP}}^{2}$, and $\tilde{\chi}_{\mathrm{VDP}}^{2}$ themselves, $\tilde{\beta}_{\mathrm{SBP}}^{2}$, and $\tilde{\beta}_{\mathrm{VDP}}^{2}$. For other GCs, only the models containing the best-fitting snapshot are shown, together with the same quantities (notably, the "best fit" in these cases is not considered a "good fit").

\section{ORCID iDs}

Nicholas Z. Rui (1) https://orcid.org/0000-0002-1884-3992

Kyle Kremer (i) https://orcid.org/0000-0002-4086-3180

Newlin C. Weatherford (iD https://orcid.org/0000-00029660-9085

Sourav Chatterjee (ib https://orcid.org/0000-0002-3680-2684

Frederic A. Rasio (1) https://orcid.org/0000-0002-7132-418X

Carl L. Rodriguez (iD https://orcid.org/0000-0003-4175-8881

Claire S. Ye (iD https://orcid.org/0000-0001-9582-881X

\section{References}

Aarseth, S., \& Heggie, D. 1998, MNRAS, 297, 794

Allard, F., Hauschildt, P., Miller, S., \& Tennyson, J. 1994, ApJL, 426, L39

Bae, Y.-B., Kim, C., \& Lee, H. M. 2014, MNRAS, 440, 2714

Baraffe, I., Chabrier, G., Allard, F., \& Hauschildt, P. 1995, ApJL, 446, L35

Baumgardt, H. 2001, MNRAS, 325, 1323

Baumgardt, H., He, C., Sweet, S. M., et al. 2019a, MNRAS, 488, 5340

Baumgardt, H., \& Hilker, M. 2018, MNRAS, 478, 1520

Baumgardt, H., Hilker, M., Sollima, A., \& Bellini, A. 2019b, MNRAS, 482, 5138

Baumgardt, H., Sollima, A., \& Hilker, M. 2020, PASA, 37, e046

Bellazzini, M., Pecci, F. F., Ferraro, F. R., et al. 2001, AJ, 122, 2569

Biggs, J., Bailes, M., Lyne, A., Goss, W., \& Fruchter, A. 1994, MNRAS, 267, 125

Breivik, K., Coughlin, S., Zevin, M., et al. 2020, ApJ, 898, 71

Cardelli, J. A., Clayton, G. C., \& Mathis, J. S. 1989, ApJ, 345, 245

Casagrande, L., \& VandenBerg, D. A. 2014, MNRAS, 444, 392

Chatterjee, S., Fregeau, J. M., Umbreit, S., \& Rasio, F. A. 2010, ApJ, 719, 915

Chatterjee, S., Umbreit, S., Fregeau, J. M., \& Rasio, F. A. 2013, MNRAS, 429, 2881

Chen, C., \& Chen, W. 2010, ApJ, 721, 1790

Chen, X., \& Han, Z. 2004, MNRAS, 355, 1182

Cohn, H. N., Lugger, P. M., Couch, S. M., et al. 2010, ApJ, 722, 20

Cool, A. M., Grindlay, J. E., Cohn, H. N., Lugger, P. M., \& Slavin, S. D. 1995 , ApJ, 439, 695

Cool, A. M., Piotto, G., \& King, I. R. 1996, ApJ, 468, 655

Dieball, A., Rasekh, A., Knigge, C., Shara, M., \& Zurek, D. 2017, MNRAS, 469,267

Fabbiano, G. 2006, ARA\&A, 44, 323

Ferraro, F., Lanzoni, B., Dalessandro, E., et al. 2012, Natur, 492, 393

Ferraro, F., Lanzoni, B., Raso, S., et al. 2018a, ApJ, 860, 36
Ferraro, F., Mucciarelli, A., Lanzoni, B., et al. 2018b, ApJ, 860, 50

Fregeau, J. M., Cheung, P., Portegies Zwart, S., \& Rasio, F. A. 2004, MNRAS, 352,1

Fregeau, J. M., Gürkan, M., Joshi, K., \& Rasio, F. 2003, ApJ, 593, 772

Fregeau, J. M., \& Rasio, F. A. 2007, ApJ, 658, 1047

Gieles, M., Balbinot, E., ISM Yaaqib, R., et al. 2018, MNRAS, 473, 4832

Gieles, M., Erkal, D., Antonini, F., Balbinot, E., \& Peñarrubia, J. 2021, arXiv:2102.11348

Giersz, M. 1998, MNRAS, 298, 1239

Giersz, M., \& Heggie, D. C. 2009, MNRAS, 395, 1173

Giersz, M., \& Heggie, D. C. 2011, MNRAS, 410, 2698

Giersz, M., Heggie, D. C., Hurley, J. R., \& Hypki, A. 2013, MNRAS, 431,2184

Giersz, M., Leigh, N., Hypki, A., Lützgendorf, N., \& Askar, A. 2015, MNRAS, 454, 3150

Glebbeek, E., Pols, O. R., \& Hurley, J. R. 2008, A\&A, 488, 1007

Green, E., \& Norris, J. E. 1990, ApJL, 353, L17

Grindlay, J., Heinke, C., Edmonds, P., Murray, S., \& Cool, A. 2001, ApJL, 563, L53

Han, M., Chun, S.-H., Choudhury, S., et al. 2017, JASS, 34, 83

Hansen, B. M., Anderson, J., Brewer, J., et al. 2007, ApJ, 671, 380

Harris, C. R., Millman, K. J., \& van der Walt, S. J. 2020, Natur, 585, 357

Harris, W. E. 1996, AJ, 112, 1487

Heggie, D. C., \& Giersz, M. 2008, MNRAS, 389, 1858

Heggie, D. C., \& Giersz, M. 2014, MNRAS, 439, 2459

Hénon, M. 1971, in IAU Coll. 10, Gravitational N-Body Problem (Cambridge: Cambridge Univ. Press), 151

Hui, C., Cheng, K., \& Taam, R. E. 2010, ApJ, 714, 1149

Hunter, J. D. 2007, CSE, 9, 90

Hurley, J. R., Pols, O. R., \& Tout, C. A. 2000, MNRAS, 315, 543

Hurley, J. R., Tout, C. A., \& Pols, O. R. 2002, MNRAS, 329, 897

Hut, P., \& Heggie, D. 2003, The Gravitational Million-Body Problem (Cambridge: Cambridge Univ. Press)

Hut, P., McMillan, S., Goodman, J., et al. 1992, PASP, 104, 981

Ivanova, N., Chaichenets, S., Fregeau, J., et al. 2010, ApJ, 717, 948

Ivanova, N., Heinke, C., Rasio, F., et al. 2006, MNRAS, 372, 1043

Jones, E., Oliphant, T., Peterson, P., et al. 2001, SciPy: Open Source Scientific Tools for Python, http://www.scipy.org/

Jonker, P., Méndez, M., Nelemans, G., Wijnands, R., \& Van Der Klis, M. 2003, MNRAS, 341, 823

Joshi, K. J., Nave, C. P., \& Rasio, F. A. 2001, ApJ, 550, 691

Joshi, K. J., Rasio, F. A., \& Zwart, S. P. 2000, ApJ, 540, 969

Kacharov, N., Bianchini, P., Koch, A., et al. 2014, A\&A, 567, A69

Kaluzny, J., \& Krzeminski, W. 1993, MNRAS, 264, 785

Kamann, S., Husser, T.-O., Dreizler, S., et al. 2017, MNRAS, 473, 5591

Knigge, C. 2012, MmSAI, 83, 549

Kong, A. K., Bassa, C., Pooley, D., et al. 2006, ApJ, 647, 1065

Kremer, K., Chatterjee, S., Breivik, K., et al. 2018a, PhRvL, 120, 191103

Kremer, K., Chatterjee, S., Rodriguez, C. L., \& Rasio, F. A. 2018b, ApJ, 852, 29

Kremer, K., Chatterjee, S., Ye, C. S., Rodriguez, C. L., \& Rasio, F. A. 2019, ApJ, 871, 38 
Kremer, K., Claire, S. Y., Rui, N. Z., et al. 2020, ApJS, 247, 48

Kremer, K., Ye, C. S., Chatterjee, S., Rodriguez, C. L., \& Rasio, F. A. 2018, ApJL, 855, L15

Kremer, K., Ye, C. S., Chatterjee, S., Rodriguez, C. L., \& Rasio, F. A. 2020, in Proc. IAU Vol. 351, Star Clusters: From the Milky Way to the Early Universe, ed. A. Bragaglia et al. (San Francisco, CA: ASP), 357

Kruijssen, J. D., Pfeffer, J. L., Reina-Campos, M., Crain, R. A., \& Bastian, N. 2019, MNRAS, 486, 3180

Lorimer, D. R. 2008, LRR, 11, 8

Lynch, R. S., Freire, P. C., Ransom, S. M., \& Jacoby, B. A. 2012, ApJ, 745,109

Mackey, A., \& Gilmore, G. 2003a, MNRAS, 338, 85

Mackey, A., \& Gilmore, G. 2003b, MNRAS, 338, 120

Mackey, A., \& Gilmore, G. 2003c, MNRAS, 340, 175

Mackey, A., Wilkinson, M., Davies, M. B., \& Gilmore, G. 2008, MNRAS, 386, 65

Maoz, D., Mannucci, F., \& Nelemans, G. 2014, ARA\&A, 52, 107

McKinney, W. 2010, in Proc. 9th Python in Science Conf., Data Structures for Statistical Computing in Python, ed. S. van der Walt \& J. Millman (Chicago, IL: SciPy), 51

McLaughlin, D. E., \& van der Marel, R. P. 2005, ApJS, 161, 304

Milone, A., Piotto, G., Bedin, L., et al. 2012, A\&A, 540, A16

Noyola, E., \& Gebhardt, K. 2006, AJ, 132, 447

Oliphant, T. E. 2006, CSE, 9, 10

Paresce, F., De Marchi, G., \& Romaniello, M. 1995, ApJ, 440, 216

Pattabiraman, B., Umbreit, S., Liao, W.-k., et al. 2013, ApJS, 204, 15

Perera, B., Stappers, B., Lyne, A., et al. 2017, MNRAS, 468, 2114

Pérez, F., \& Granger, B. E. 2007, CSE, 9, 21

Peuten, M., Brockamp, M., Kuepper, A. H., \& Kroupa, P. 2014, ApJ, 795, 116

Piatti, A. E. 2018, MNRAS, 473, 492

Pooley, D., Lewin, W. H., Anderson, S. F., et al. 2003, ApJL, 591, L131

Portegies Zwart, S. F., McMillan, S. L., \& Gieles, M. 2010, ARA\&A, 48, 431

Robinson, E. L. 1976, ARA\&A, 14, 119
Rodrigo, C., \& Solano, E. 2013, IVOA Note 2013 May 10, Filter Profile Service Access Protocol Version 1.0, http://ivoa.net/documents/Notes/ SVOFPSDAL/index.htmlGoogle Scholar

Rodrigo, C., Solano, E., \& Bayo, A. 2020, Contributions to the XIV.0 Scientific Meeting (virtual) of the SEA, The SVO Filter Profile Service, 182

Rodriguez, C. L., Amaro-Seoane, P., Chatterjee, S., \& Rasio, F. A. 2018, PhRvL, 120, 151101

Rodriguez, C. L., Morscher, M., Wang, L., et al. 2016, MNRAS, 463, 2109

Roh, D.-G., Lee, Y.-W., Joo, S.-J., et al. 2011, ApJL, 733, L45

Rui, N. Z., Kremer, K., Weatherford, N. C., et al. 2021, cmctoolkit v1.0, Zenodo, doi:10.5281/zenodo. 4579950

Sarajedini, A., \& Demarque, P. 1990, ApJ, 365, 219

Servillat, M., Webb, N., \& Barret, D. 2008, A\&A, 480, 397

Sohn, S. T. 2018, Instrument Science Report, STIS 2018-2

Sollima, A., \& Baumgardt, H. 2017, MNRAS, 471, 3668

Stodolkiewicz, J. 1986, AcA, 36, 19

Tam, P., Kong, A., Hui, C., et al. 2011, ApJ, 729, 90

Taylor, J., Grindlay, J., Edmonds, P., \& Cool, A. 2001, ApJL, 553, L169

Trager, S., King, I. R., \& Djorgovski, S. 1995, AJ, 109, 218

The Astropy Collaboration, Robitaille, T. P., Tollerud, E. J., et al. 2013, A\&A, 558, A33

Umbreit, S., Fregeau, J. M., Chatterjee, S., \& Rasio, F. A. 2012, ApJ, 750, 31

Wang, L., Spurzem, R., Aarseth, S., et al. 2016, MNRAS, 458, 1450

Watkins, L. L., van der Marel, R. P., Bellini, A., \& Anderson, J. 2015, ApJ, 803, 29

Weatherford, N. C., Chatterjee, S., Kremer, K., \& Rasio, F. A. 2020, ApJ, 898, 162

Weatherford, N. C., Chatterjee, S., Rodriguez, C. L., \& Rasio, F. A. 2018, ApJ, 864,13

Weatherford, N. C., Fragione, G., Kremer, K., et al. 2021, ApJL, 907, L25

Virtanen, P., Gommers, R., Oliphant, T. E., et al. 2020, NatMe, 17, 261

Ye, C. S., Kremer, K., Chatterjee, S., Rodriguez, C. L., \& Rasio, F. A. 2019, ApJ, 877, 122

Zonoozi, A. H., Haghi, H., Küpper, A. H., et al. 2014, MNRAS, 440, 3172

Zonoozi, A. H., Küpper, A. H., Baumgardt, H., et al. 2011, MNRAS, 411, 1989 